GEOLOGICAL SURVEY CIRCULAR 819-A

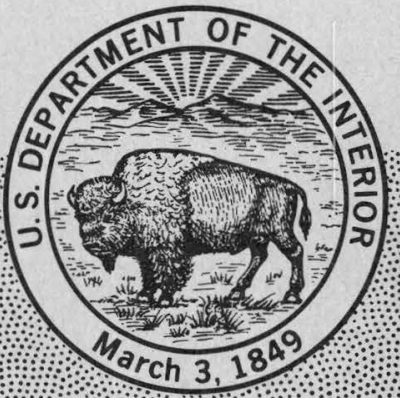

Earthquakes

in the United States,

January-March 1978 



\section{Earthquakes}

\section{in the United States, \\ January-March 1978}

By C. W. Stover, J. H. Minsch, and B. G. Reagor
GEOLOGICAL SURVEY CIRCULAR 819-A 


\section{United States Department of the Interior}

\section{CECIL D. ANDRUS, Secretary}

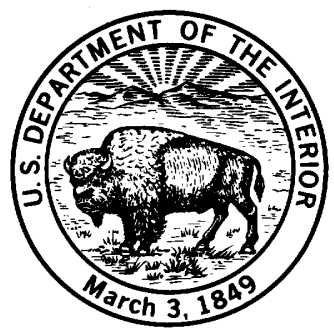

\section{Geological Survey}

H. William Menard, Director 
FIGURE 1. "Earthquake Report" form............................. Page

2. Map showing standard time zones of the conterminous United States.... 4

3. Map showing standard time zones of Alaska and Hawaii............ 5

4. Map of earthquake epicenters in the conterminous United States for

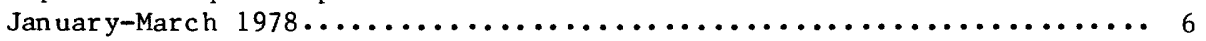

5. Map of earthquake epicenters in Alaska for January-March 1978....... 7

6. Map of earthquake epicenters in Hawaii for January-March $1978 \ldots \ldots . . .8$

7. Isoseismal map for the northern California earthquake of 26 March

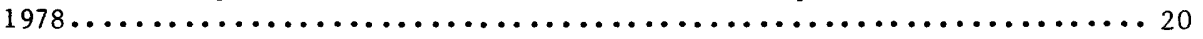

8. Isoseismal map for the Puget Sound, Washington earthquake of 11 March 1978.......................................... 24

9. Isoseismal map for the Puget Sound, Washington earthquake of

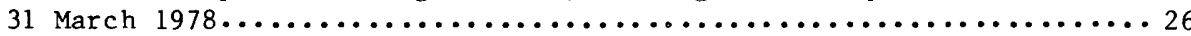

TABLES

TABLE 1. Summary of U.S. earthquakes for January-March 1978:

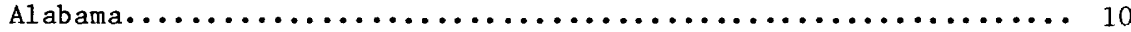

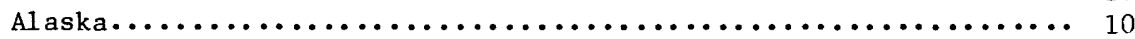

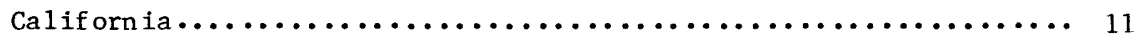

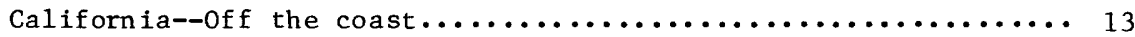

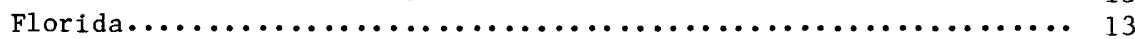

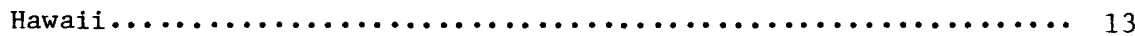

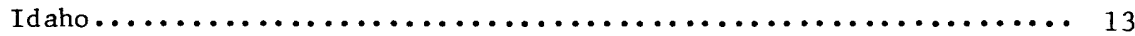

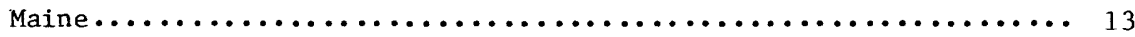

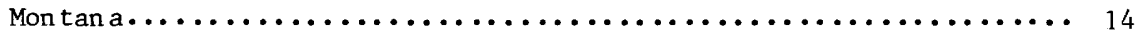

Nevada.............................................. 14

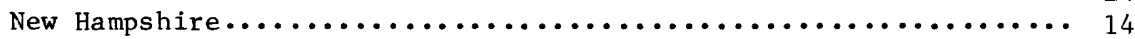

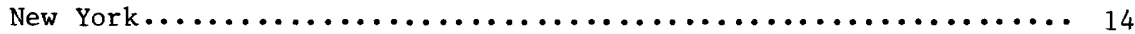

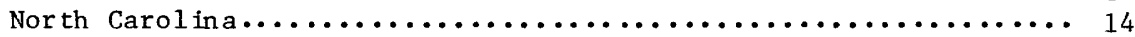

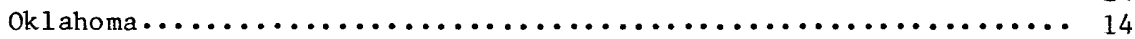

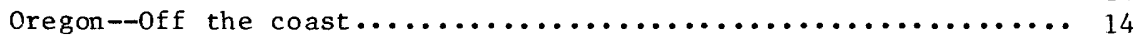

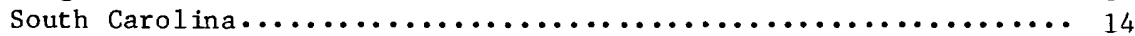

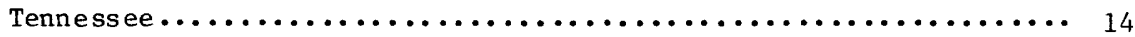

Texas.......................................... 14

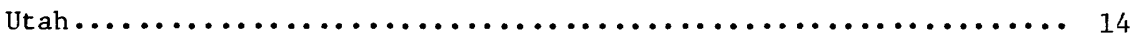

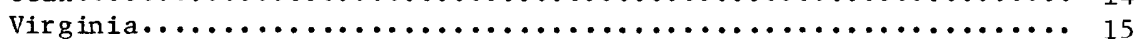

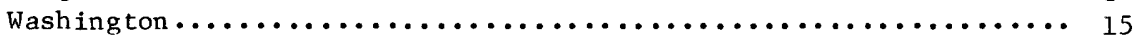

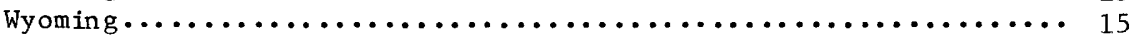


2. Summary of macroseismic data for U.S. earthquakes, January-March 1978:

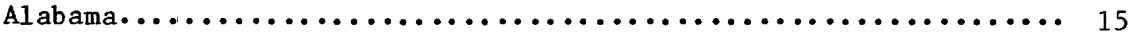

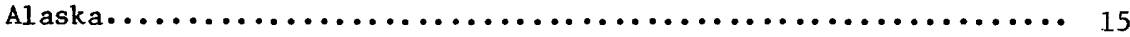

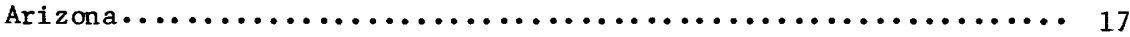

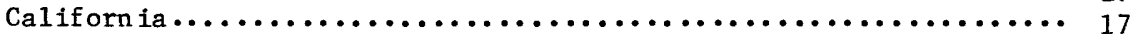

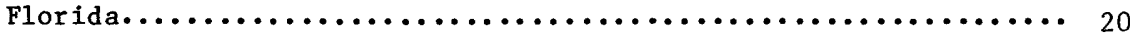

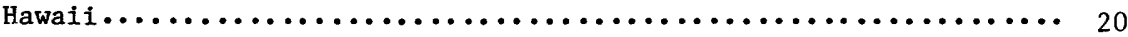

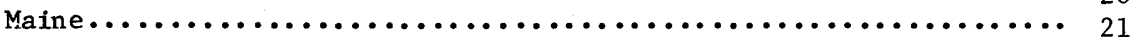

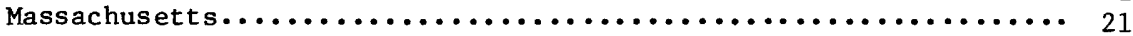

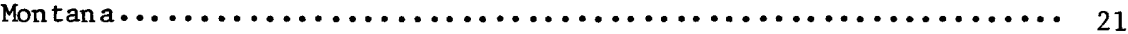

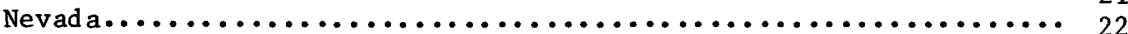

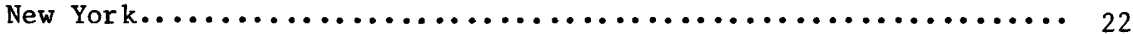

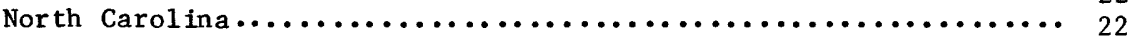

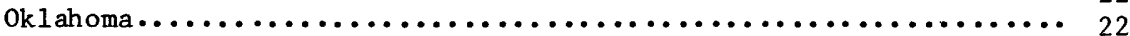

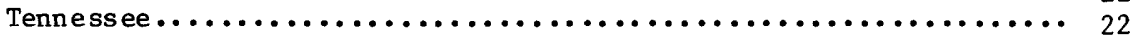

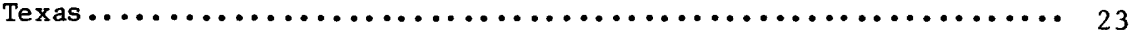

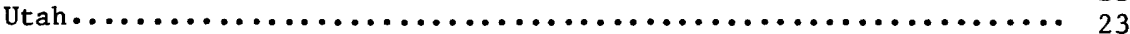

Virginia....................................... 23

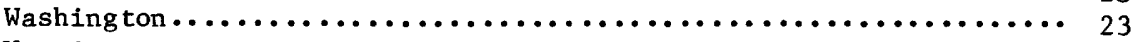

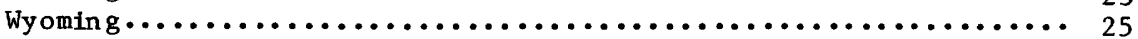




\title{
Earthquakes in the United States, January-March 1978
}

\author{
By C. W. Stover, J. H. Minsch, and B. G. Reagor
}

\section{INTRODUCTION}

The earthquake information in this publication supplements that published in the NEIS (National Earthquake Information Service) publications, PDE ("Preliminary Determination of Epicenters") and "Preliminary Determination of Epicenters, Monthly Listing," to the extent of providing detailed felt and intensity data, as well as isoseismal maps for U.S. earthquakes. The purpose is to provide a complete listing of macroseismic effects of earthquakes, which $c$ an be used in risk studies, nuclear power plant site evaluations, seismicity studies, and answering inquiries by the public.

This publication contains two major sections. The first (table 1) is a tabular listing of earthquakes in chronological order by State, consisting of the following basic information: date, origin time, hypocenter, magnitude, maximum intensity, and computational source of the hypocenter. The second section consists of three maps and table 2, which lists detailed intensity information. The list of earthquakes in table 1 was compiled from those located in Alaska or off the coasts that were published in the PDE; from hypocenters located in the conterminous United States using the U.S. Geological Survey program SEDAS; from hypocenters in California above magnitude 3.0 , supplied by California Institute of Technology, Pasadena, the University of California, Berkeley, and other offices of the U.S. Geological Survey; from hypocenters in Hawaii supplied by the Hawaiian Volcano Observatory; and from any others that were felt or that caused damage, regardless of magnitude or availability of a hypocenter. Known or suspected explosions are also listed.

The intensities and macroseismic data were compiled from information obtained through questionnaires, from newspaper articles, and with the cooperation of other Government agencies, State institutions, local organizations, and individuals. (See "Acknowledgments" for a list of collaborators.) Figure 1 is the questionnaire in current use by the NEIS. Other versions of this questionnaire are used by state agencies, engineering firms, and other Government agencies to collect intensity data. Anyone wishing to submit felt or damage information on earthquakes for inclusion in future reports should send it to the National Earthquake Information Service, Stop 967, Box 25046, Denver Federal Center, Denver, CO 80225. Copies of the current "Earthquake Report" questionnaire can be obtained at this address.

The primary method used by the NEIS to
collect macroseismic information is a questionnaire canvass using the "Earthquake Report" forms, which are mailed to postmasters in the area affected by the earthquake. The postmasters complete the forms and return them to the NEIS, where they are evaluated and an intensity value is assigned. The intensity observations are mapped and contoured by isoseismals. Isoseismal contours present a generalization of intensity data and an extrapolation of these data to regions from which there are no observations; they do not necessarily account for every individual observation.

The data in table 2 will be included in the "Earthquake Description" section of "United States Earthquakes," an annual publication, to which later data from other sources may be added for the purpose of updating and completeness. "United States Earthquakes" is published jointly by the U.S. Geological Survey, Department of the Interior, and the Environmental Data Service, National Oceanic and Atmospheric Administration, Department of Commerce.

\section{DISCUSSION OF TABLES}

The parameters for the earthquakes in table 1 and table 2 include the date, origin time, hypocenter (epicenter and focal depth), magnitude, in tensity, and hypocenter source. The origin time and date are listed in Universal Coordinated Time (UTC) and local standard time based on the time-zone maps in figures 2 and 3 . The epicenters, which were taken from those published in the PDE, or from other sources as noted, are listed here to two decimals. The accuracy of the 
Please answer this questionnaire and return as soon as possible

1. Was an earthquake felt by anyone in your town near the date and time indicated on the opposite page?

$\begin{array}{llll}\text { No: } & \text { Please refold and tape for return mail. } & \\ \text { Yes: Date } & \text { Time } & \text { AM } & \text { Standard time } \\ & & \text { PM } & \text { Daylight time }\end{array}$

Name of person filling out form

$\begin{array}{ll}\text { Address } & \\ \text { City } & \text { County } \\ \text { State } & \text { Zip code }\end{array}$

If you felt the earthquake, complete the following section. If others felt the earthquake but you did not, skip the personal report and complete the community report.

PERSONAL REPORT

2a. Did you personally feel the earthquake? 1 Yes No

b. Were you awakened by the earthquake? 2 Yes No

c. Were you frightened by the earthquake? 3 Yes No

d. Were you at 4 Home 5 Work 6 Other?

e. Town and zip code of your location at time of earthquake

f. Check your activity when the earthquake occurred:

\begin{tabular}{|c|c|c|c|c|c|c|c|}
\hline$t$ & Walking & 8 & Sleeping & 9 & Lying down & 10 & Standing \\
\hline 11 & Driving (car in motion) & 12 & Sitting & 13 & Other & & \\
\hline
\end{tabular}

g. Were you

h. If inside, on what floor were you?

14 Inside or 15 Outside?

i. Vibration could be described as 17 Light 18 Heavy

j. Was there earth noise? No 19 Faint 20 Moderate 21 Loud

k. Direction of noise North South 20 East

1. Estimated duration of 22 Sudden, sharp (less than 10 secs) 23 Long (30-60 secs) shaking 24 Short (10-30 secs)

Continue on to next section which should include personal as well as reported observations.

Town and zip code

$$
\text { COMMUNITY REPORT }
$$

DO NOT INCLUDE EFFECTS FROM OTHER COMMUNITIES/TOWNS

Check one box for each question that is applicable.

$\begin{array}{ccccccccccc}\text { 3a. The earthquake was felt by } & \text { No one } 25 & \text { Few } & 26 & \text { Several } & 27 & \text { Many } & 28 & \text { All? } \\ \text { b. This earthquake awakened } & \text { No one } 29 & \text { Few } & 30 & \text { Several } & 31 & \text { Many } & 32 & \text { All? } \\ \text { c. This earthquake frightened } & \text { No one } 33 & \text { Few } & 34 & \text { Several } & 35 & \text { Many } & 36 & \text { All? }\end{array}$

4. What indoor physical effects were noted in your community?

Windows, doors, dishes rattled 37 Yes No

Buildings creaked

Building trembled (shook)

Hanging pictures (more than one) 41 Swung 42 Out of place 43 Fallen

Liquid in small containers 44 Spilled 45 Slightly disturbed

Windows 46 Few cracked 47 Some broken 48 Many broken

Were small objects (dishes, knick-knacks, lamps) $\quad$ Unmoved 49 Moved

50 Overturned 51 Broken?

Were light furniture or small appliances Unmoved $\quad 52$ Moved

$\begin{array}{lllll} & & \text { Overturned } & 54 & \text { Damaged seri } \\ \text { Were heavy furniture or appliances } & \text { Unmoved } & 55 & \text { Overturned }\end{array}$ 56 Moved 57 Damaged seriously,

Did hanging objects ordoors swing? 58 Slightly 59 Moderately 60 Violently

Can you estimate direction? North/South East/West Other

$\begin{array}{llllll}\text { Pendulum clocks } & 61 & \text { Stopped } 62 & \text { Started } 63 \text { Faster or slower }\end{array}$

Continued on the reverse side

FIGURE 1.-Example of the "Earthquake Report" form used for evaluating the intensities of earthquakes. A, front side. 
5. Indicate effects of the following types to interior walls if any:
Plaster/stucco 64 Large cracks
65 Fell in large amounts
Dry wall
66 Large cracks
67 Fell in large amounts
Ceiling tiles
68 Large cracks
69 Fell in large amounts

6. What outdoor physical effects were noted in your community?

\begin{tabular}{|c|c|c|c|c|c|c|}
\hline Trees and bushes shaken & 70 & Slightiy & 71 & Moderately & 72 & Strongly \\
\hline Standing vehicles rocked & 73 & Slightly & 74 & Moderately & & \\
\hline Moving vehicles rocked & 75 & Slightly & 76 & Moderately & & \\
\hline Water splashed onto sides of & & & & & & \\
\hline lakes, ponds, swimming pools & & 77 Yes & & No & & \\
\hline Elevated water tanks & 78 & Cracked & 79 & Twisted & $\begin{array}{l}80 \\
\text { (thr }\end{array}$ & $\begin{array}{l}\text { Fallen } \\
\text { rown down) }\end{array}$ \\
\hline Industrial cooling units & 81 & Displaced & 82 & Rotated & 83 & Fallen \\
\hline Tombstones & $\begin{array}{l}84 \\
87\end{array}$ & $\begin{array}{l}\text { Displaced } \\
\text { Fallen }\end{array}$ & 85 & Cracked & 86 & Rotated \\
\hline Chimneys & $\begin{array}{l}88 \\
91\end{array}$ & $\begin{array}{l}\text { Bricks loosene } \\
\text { Broken at roof }\end{array}$ & f line & $\begin{array}{r}89 \quad \text { Twisted } \\
92\end{array}$ & $\begin{array}{c}90 \\
\text { Bricks }\end{array}$ & $\begin{array}{l}\text { Fallen } \\
\text { fallen }\end{array}$ \\
\hline Railroad tracks bent & 93 & Slightly & 94 & Greatly & & \\
\hline Stone or brick fences /walls & 95 & Open cracks & 96 & Fallen & 97 & Destroyed \\
\hline Underground pipes & 98 & Broken & 99 & Out of service & & \\
\hline Highways or streets & 100 & Large cracks & & Large disp & lacemer & nts \\
\hline Sidewalks & 102 & Large cracks & & Large disp & Slaceme & ints \\
\hline
\end{tabular}

7a. Check below any structural damage to buildings.

$\begin{array}{lllll}\text { Foundation } & 104 & \text { Cracked } & 105 & \text { Destroyed } \\ \text { Interior walls } & 106 & \text { Split 107 Fallen } & 108 & \text { Separated from ceiling or floor } \\ \text { Exterior walls } & 109 & \text { Large Cracks } & 110 & \text { Bulged outward } \\ & 111 & \text { Partial collapse } & 112 & \text { Total collapse } \\ \text { Building } & 113 & \text { Moved on foundation } & 114 & \text { Shifted off foundation }\end{array}$

b. What type of construction was the building that showed this damage?
115 Wood
116 Stone
117 Brick veneer
118 Other
119 Brick 120 Cinderblock
121 Reinforced concrete
122 Mobile home

c. What was the type of ground under the building?

$\begin{array}{lllllll}\text { Don't know } & 123 & \text { Sandy soil } & 124 & \text { Marshy } & 125 \text { Fill } \\ 126 \text { Hard rock } & 127 & \text { Clay soil } & 128 & \text { Sandstone, limestone, shale } \\ \text { the ground: } & 129 & \text { Level } & 130 & \text { Sloping } & 131 \text { Steep? }\end{array}$

d. Was the ground:

129 Level

130 Sloping 131 Steep?

e. Check the approximate age of the building: 132 Built before $1935 \quad 133$ Built 1935-65 134 Built after 1965

8. Check below any structural damage to

$\begin{array}{clllllll}\text { Bridges/Overpasses } & 135 & \text { Concrete } & 136 & \text { Wood } 137 & \text { Steel } & 138 & \text { Other } \\ \text { Damage was } & 139 & \text { Slight } & 140 & \text { Moderate } & 141 & \text { Severe } \\ \text { Dams } & 142 & \text { Concrete } & 143 & \text { Large earthen } & & \\ \text { Damage was } & 144 & \text { Slight } & 145 & \text { Moderate } & 146 & \text { Severe }\end{array}$

9. What geologic effects were noted in your community?

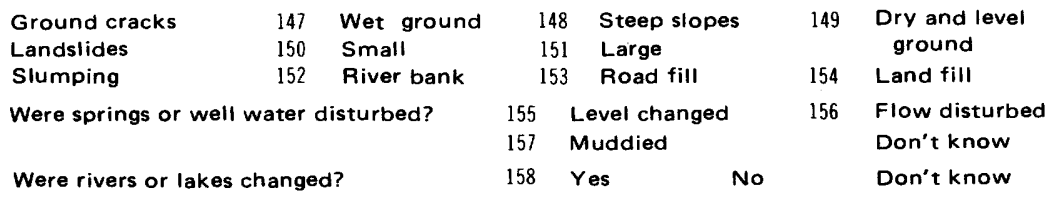

10a. What percentage of buildings were damaged? Within 2 city blocks of your location $\quad$ None 159 Few (about 5\%)

b. In area covered by your zip code

$\begin{array}{llll}160 & \text { None } & 159 & \text { Few (about 5\%) } \\ \text { Many (about 50\%) } & 161 & \text { Most (about 75\%) } \\ \text { None } & 162 & \text { Few (about 5\%) } \\ 163 & \text { Many (about 50\%) } & 164 & \text { Most (about 75\%) }\end{array}$

Thank you for your time and information. Refold this card and tape for return mail.

FIGURE 1.--Example of the "Earthquake Report" form used for evaluating the intensities of earthquakes. $B$, reverse side. 


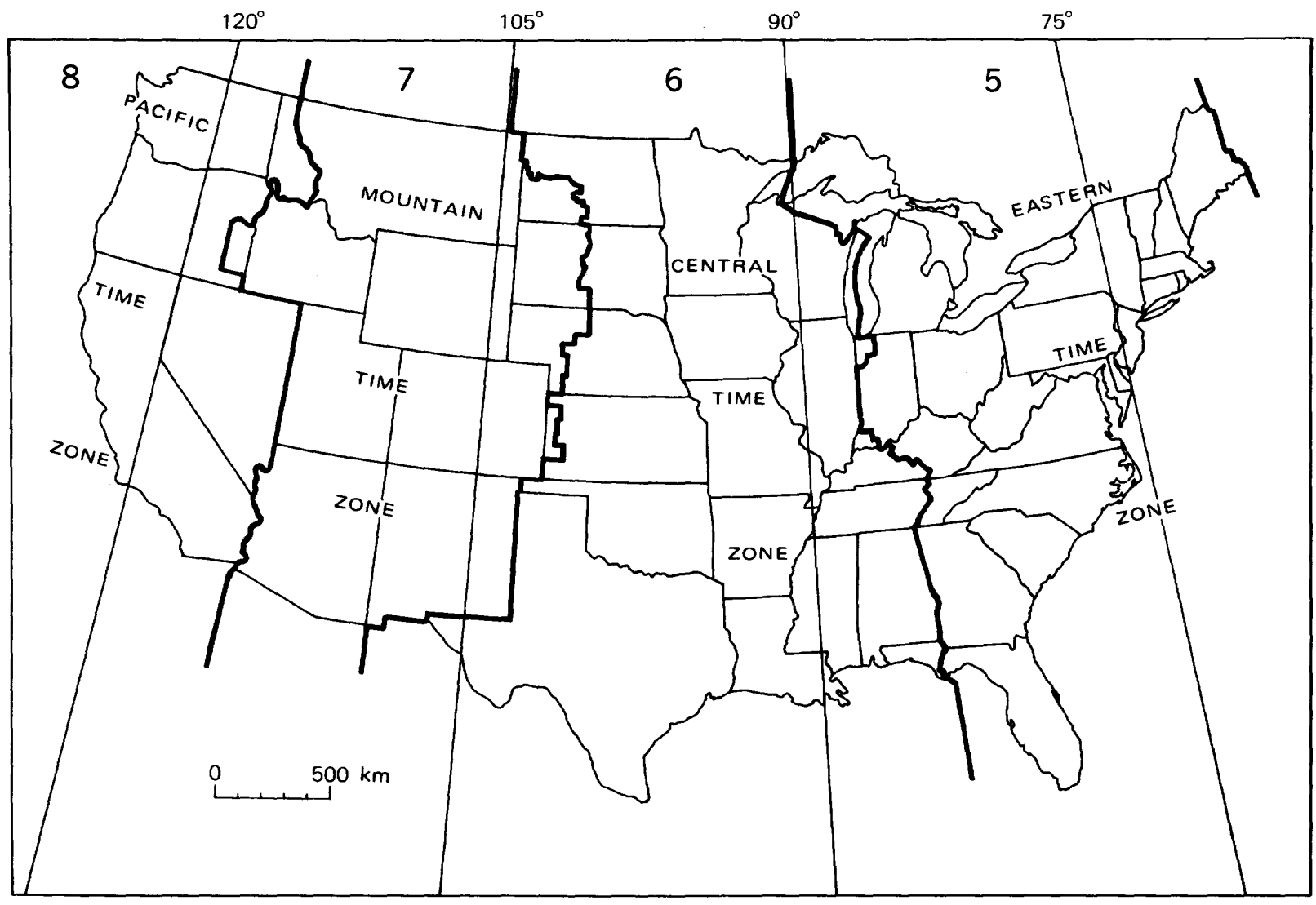

FIGURE 2.- Standard time zones of the conterminous United States. The number in each zone shows the number of hours to be subtracted from Universal Coordinated Time to convert to local standard time. (Subtract 1 hour less for local daylight-saving time.)

epicenters is that claimed by the institution supplying the hypocenter and is not necessarily the accuracy indicated by the number of decimals listed. The epicenters located by the NEIS have a varying degree of accuracy, usually two-tenths of a degree or less, depending on their continental or oceanic location. The oceanic hypocenters are less accurate than those on the continent, even though both are listed to two decimals. Depths are listed to the nearest whole kilometer.

Figures 4-6 are maps summarizing the earthquake activity for the conterminous United States, Alaska, and Hawaii for the period January-March 1978. The magnitudes plotted in these figures are based on MI or mbLg; if neither was computed, then on MS; and fin ally on mb, when it was the only magnitude computed.

The magnitude values 1 isted in tables 1 and 2 were furnished by cooperating institutions or determined by the NEIS. The computational sources are labeled according to the assigned letter codes shown in headnotes to tables 1 and 2; the letter follows the value listed under the column heading "Magnitude." In table 1 the absence of a letter code indicates that the NEIS is the source. In table 2 the magnitude source is the same as the location source unless indicated otherwise, by an alphabetic character to the right of the magnitude value. The magnitude values calculated by the NEIS are based on the following formulas:

$$
M S=\log (A / T)+1.66 \log D+3.3 \text {, }
$$

as adopted by the International Association of Seismology and Physics of the Earth's Interior (IASPEI; Bath, 1966, p. 153), where A is the maximum horizontal surface-wave ground amplitude, in micrometers; $T$ is the period, in seconds, and $18<T<22$; and $D$ is the distance, in gepcentric degrees (station to epicenter), and $20^{\circ} \leq \mathrm{D} \leq 160^{\circ}$. No depth correction is made for depths less than $50 \mathrm{~km}$.

$$
m b=\log (A / T)+Q(D, h),
$$

as defined by Gutenberg and Richter (1956), except that $T$, the period in seconds, is restricted to $0.1<\mathrm{T}<3.0$, and $\mathrm{A}$, the ground amplitude in micrometers, is not necessarily the maximum of the $P$-wave group. $Q$ is a function of distance $D$ and depth $h$, where $D \geq 5^{\circ}$.

$$
M I=\log A_{-} \log A_{0} \text {, }
$$




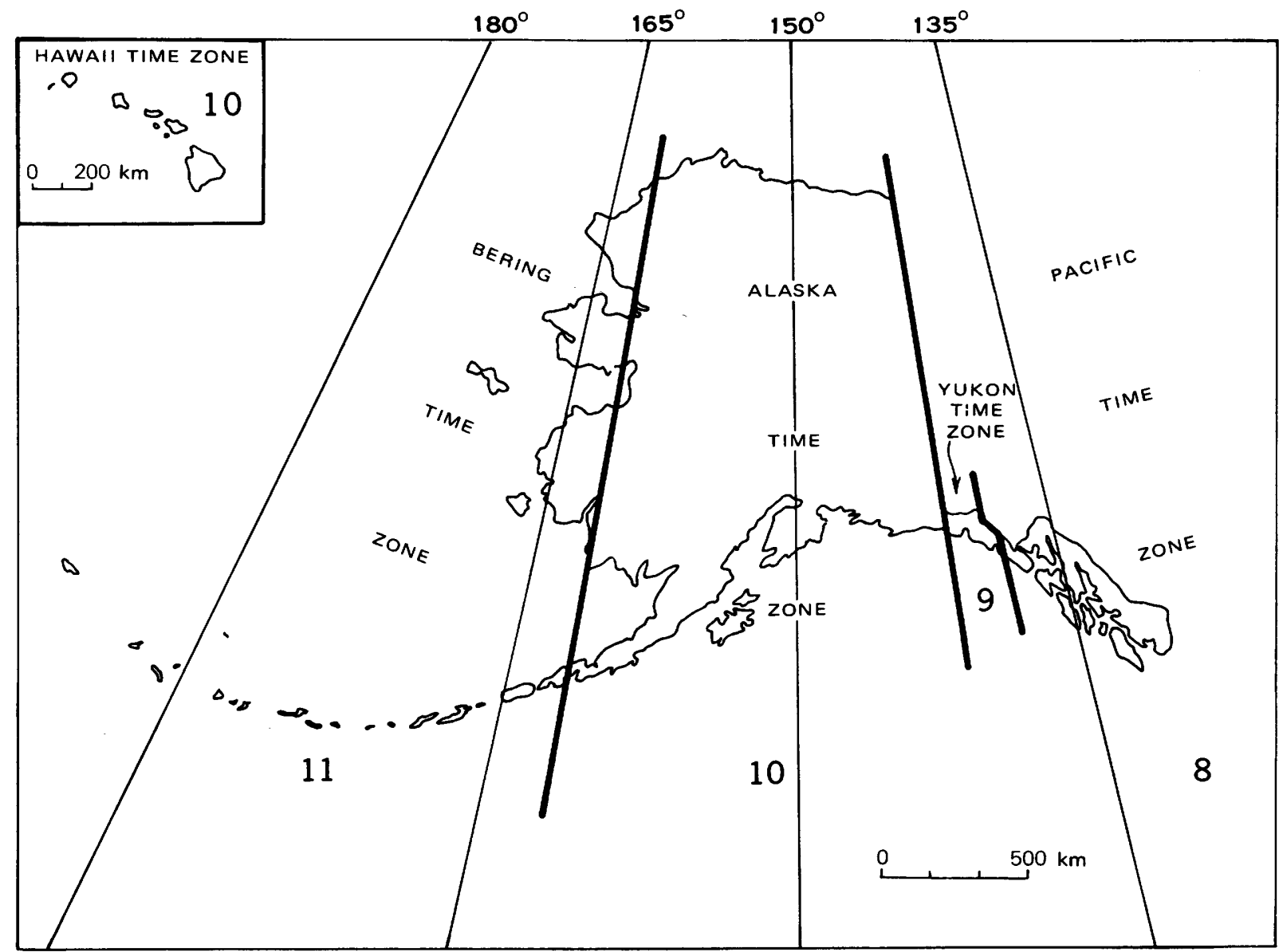

FIGURE 3.- Standard time zones of Alaska and Hawaii. The number in each zone shows the number of hours to be subtracted from Universal Coordinated Time to convert to local standard time. (Subtract 1 hour less for local daylight-saving time.)

as defined by Richter (1958, p. 340), where A is the maximum trace amplitude in millimeters, written by a Wood-Anderson torsion seismometer, and $\log A_{\circ}$ is a standard value as a function of distance, where the distance is $\leq 600 \mathrm{~km}$. MI values are also calculated from other seismometers by conversion of recorded ground motion to the expected response of the torsion seismometer.

$$
\begin{gathered}
\operatorname{mbLg}=3 \cdot 75+0.90(\log \mathrm{D})+\log (\mathrm{A} / \mathrm{T}) \\
0.5^{\circ} \leq \mathrm{D} \leq 4^{\circ}, \\
\operatorname{mbLg}=3 \cdot 30+1.66(\log \mathrm{D})+\log (\mathrm{A} / \mathrm{T}) \\
4^{\circ} \leq \mathrm{D} \leq 30^{\circ},
\end{gathered}
$$

as proposed by Nuttli (1973), where A/T is expressed in micrometers per second, calculated from the vertical-component 1 -second $\mathrm{Lg}$ waves, and $D$ is the distance in geocentric degrees.

All of the intensity values (indicated by Roman numerals) listed in this summary were derived, using the Modified Mercalli Intensity Scale of 1931 (Wood and Neumann, 1931) shown below, from the evaluation of "Earthquake Report" forms; from field reports by U.S. Geological Survey personnel, engineering firms, or universities; and from detailed macroseismic data communicated to the NEIS by people in the area affected by the earthquake. All earthquake reports received which contain minimal information are assigned an Intensity II. These reports are filed in the offices of the NEIS or in government archives and are available for detailed study.

\section{MODIFIED MERCALLI INTENSITY SCALE OF 1931}

Adapted from Sieberg's Mercalli-Cancani scale, modified and condensed.

I. Not felt - or, except rarely under especially favorable circumstances. Under certain conditions, at and outside the boundary of the area in which a great shock is felt: sometimes birds, animals, reported uneasy or disturbed; sometimes 


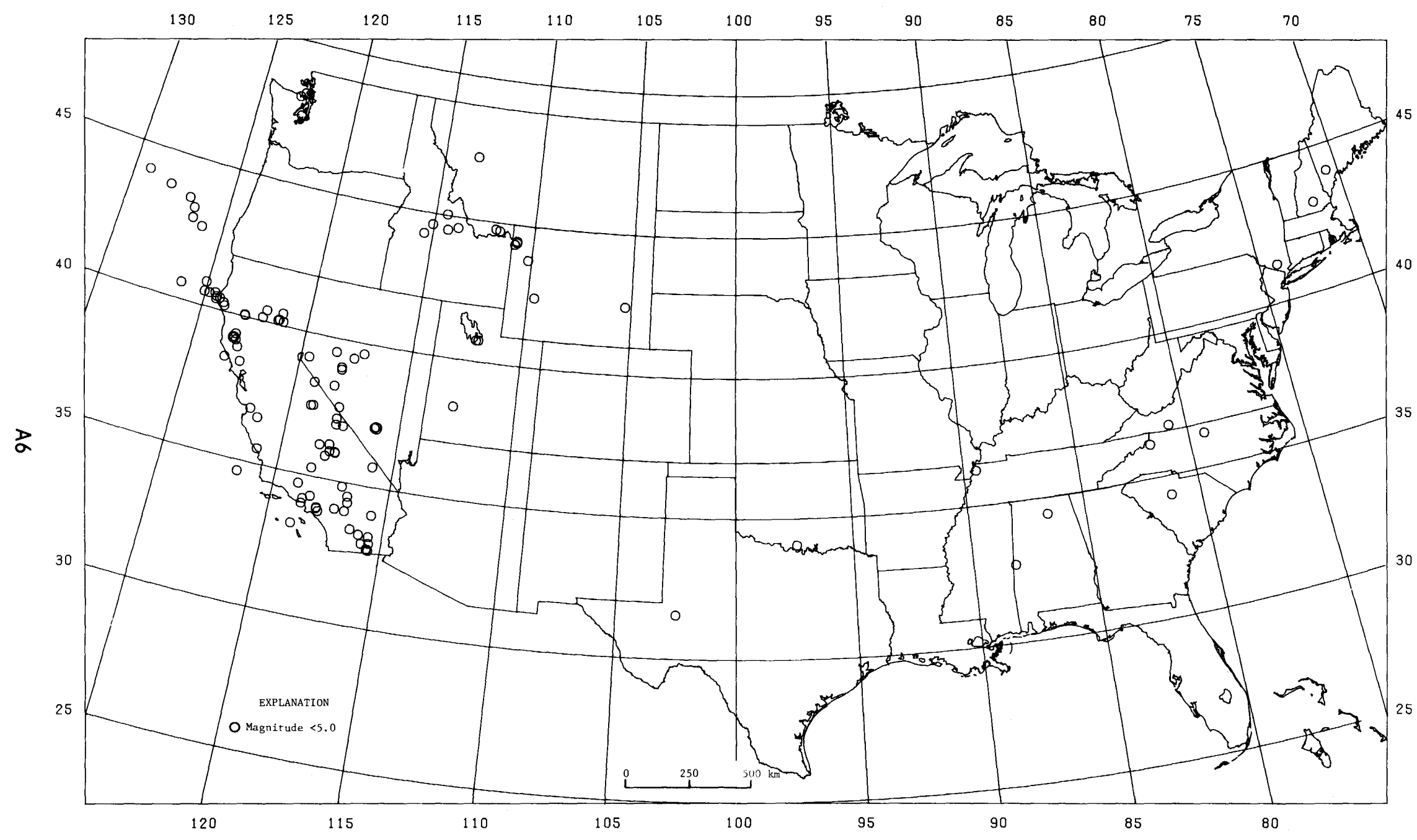

FIGURE 4.--Earthquake epicenters in the conterminous United States for January-March 1978 , plotted from table 1. 


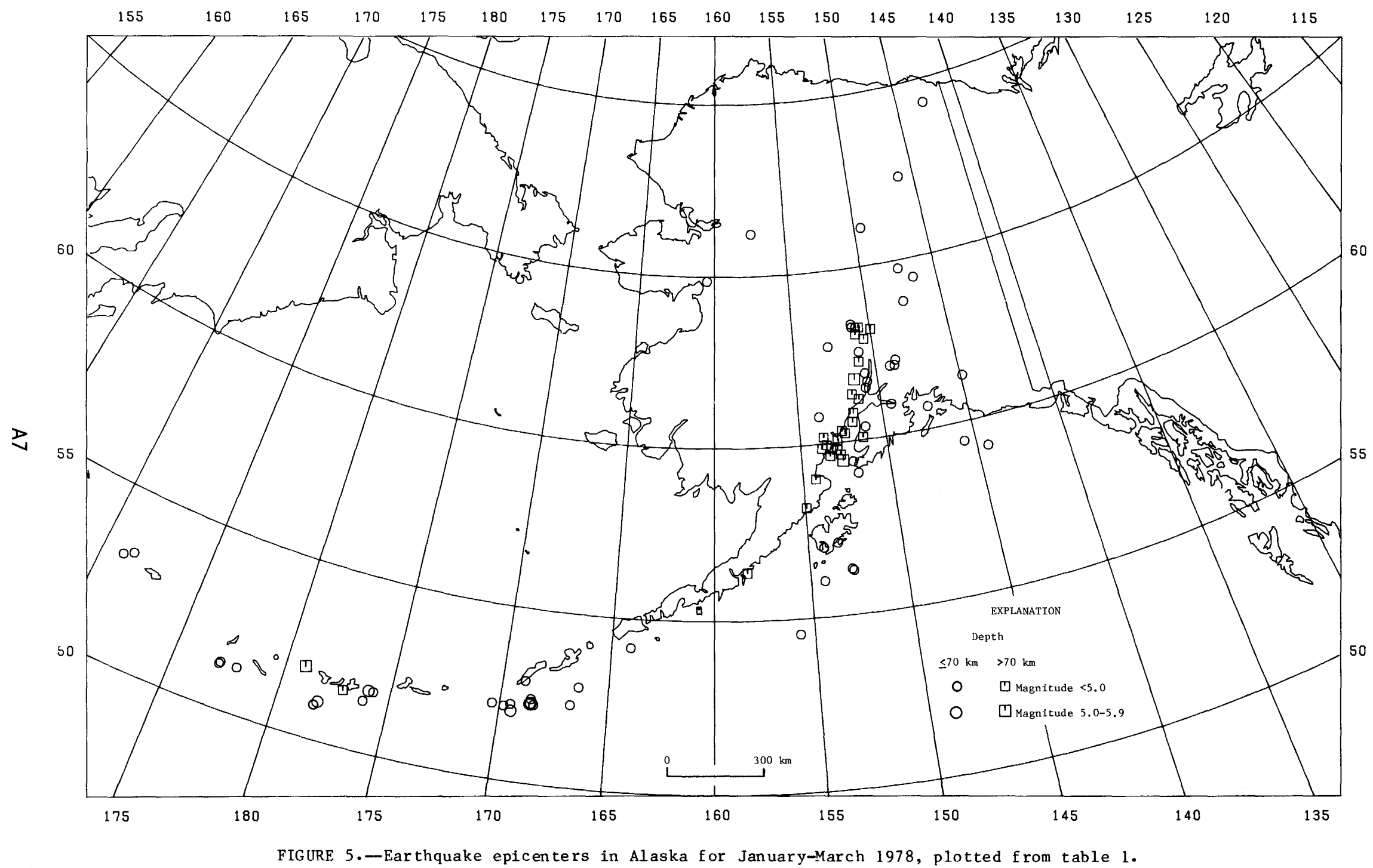




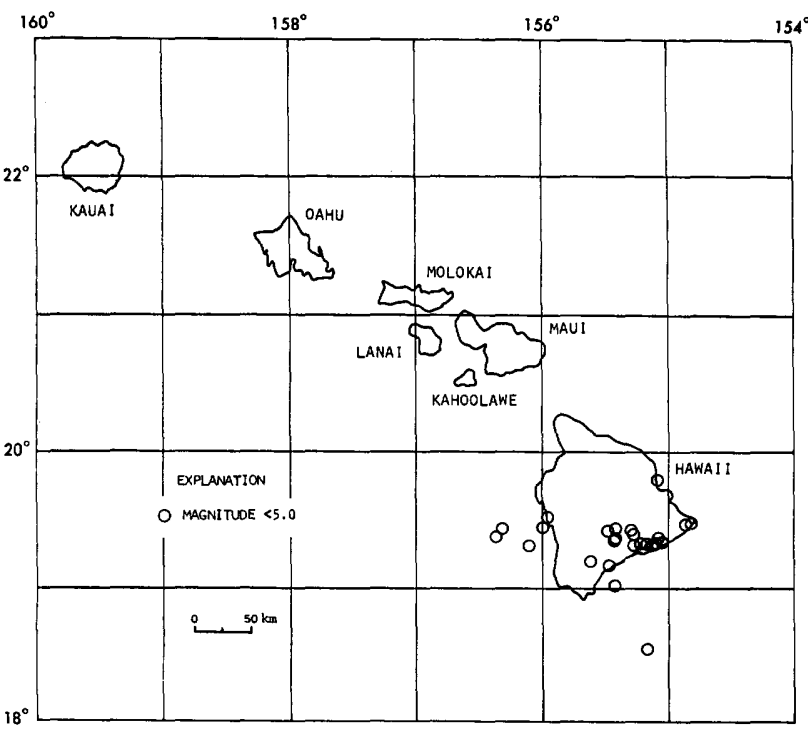

FIGURE 6.-Earthquake epicenters in Hawaii for January-March 1978, plotted from table 1 .

dizziness or nausea experienced; sometimes trees, structures, liquids, bodies of water, may sway--doors may swing, very slowly.

II. Felt indoors by few, especially on upper floors, or by sensitive, or nervous persons. Also, as in grade $I$, but often more noticeably: sometimes hanging objects may swing, especially when delicately suspended; sometimes trees, structures, liquids, bodies of water, may sway, doors may swing, very slowly; sometimes birds, animals, reported uneasy or disturbed; sometimes dizziness or nausea experienced.

III. Felt indoors by several, motion usually rapid vibration. Sometimes not recognized to be an earthquake at first. Duration estimated in some cases. Vibration like that due to passing of light, or lightly loaded trucks, or heavy trucks some distance away. Hanging objects may swing slightly. Movements may be appreciable on upper levels of tall structures. Rocked standing motor cars slightly.

IV. Felt indoors by many, outdoors by few. Awakened few, especially light sleepers. Frightened no one, unless apprehensive from previous experience. Vibration like that due to passing of heavy or heavily loaded trucks. Sensation like heavy body striking building or falling of heavy objects inside. Rattling of dishes, windows, doors; glassware and crockery clink and clash. Creaking of walls, frame, especially in the upper range of this grade. Hanging objects swung, in numerous instances. Disturbed liquids in open vessels slightly. Rocked standing motor cars noticeably.

v. Felt indoors by practically all, outdoors by many or most: outdoors direction estimated. Awakened many, or most. Frightened few--slight excitement, a few ran outdoors. Buildings trembled throughout. Broke dishes, glassware, to some extent. Cracked windows--in some cases, but not generally. Overtumed vases, small or unstable objects, in many instances, with occasional fall. Hanging objects, doors, swing generally or considerably. Knocked pictures against walls, or swung them out of place. Opened, or closed, doors, shutters, abruptly. Pendulum clocks stopped, started or ran fast, or slow. Moved small objects, fumishings, the latter to slight extent. Spilled liquids in small amounts from well-filled open containers. Trees, bushes, shaken slightly.

VI. Felt by all, indoors and outdoors. Frightened many, excitement general, some alarm, many ran outdoors. Awakened all. Persons made to move unsteadily. Trees, bushes, shaken slightly to moderately. Liquid set in strong motion. Small bells rang---church, chapel, school, etc. Damage slight in poorly built buildings. Fall of plaster in small amount. Cracked plaster somewhat, especially fine cracks chimneys in some instances. Broke dishes, glassware, in considerable quantity, also some windows. Fall of knick-knacks, books, pictures. Overturned furniture in many instances. Moved furnishings of moderately heavy kind.

VII. Frightened all--general alarm, all ran outdoors. Some, or many, found it difficult to stand. Noticed by persons driving motor cars. Trees and bushes shaken moderately to strongly. Waves on ponds, lakes, and running water. Water turbid from mud stirred up. Incaving to some extent of sand or gravel stream banks. Rang large church bells, etc. Suspended objects made to quiver. Damage negligible in buildings of good design and construction, slight to moderate in well-built ordinary buildings, considerable in poorly built or badly designed buildings, adobe houses, old walls (especially where laid up without mortar), spires, etc. Cracked chimneys to considerable extent, walls to some extent. Fall of plaster in considerable to large amount, also some stucco. Broke numerous windows, furniture to some extent. Shook down loosened brickwork and tiles. Broke weak chimneys at the roof-line (sometimes 
damaging roofs). Fall of cornices from towers and high buildings. Dislodged bricks and stones. Overturned heavy furniture, with damage from breaking. Damage considerable to concrete irrigation ditches.

VIII. Fright general--alarm approaches panic. Disturbed persons driving motor cars. Trees shaken strongly--branches, trunks, broken off, especially palm trees. Ejected sand and mud in small amounts. Changes: temporary, permanent; in flow of springs and wells; dry wells renewed flow; in temperature of spring and well waters. Damage slight in structures (brick) built especially to withstand earthquakes. Considerable in ordinary substantial buildings, partial collapse: racked, tumbled down, wooden houses in some cases; threw out panel walls in frame structures, broke off decayed piling. Fall of walls. Cracked, broke, solid stone walls seriously. Wet ground to some extent, also ground on steep slopes. Twisting, fall, of chimeys, columns, monuments, also factory stacks, towers. Moved conspicuously, overtumed, very heavy furniture.

IX. Panic general. Cracked ground conspicuously. Damage considerable in (masonry) structures built especially to withstand earthquakes: Threw out of plumb some wood-frame houses built especially to withstand earthquakes; great in substantial (masonry) buildings, some collapse in large part; or wholly shifted frame buildings off foundations, racked frames; serious to reservoirs; underground pipes sometimes broken.

X. Cracked ground, especially when loose and wet, up to widths of several inches; fissures up to a yard in width ran parallel to canal and stream banks. Landslides considerable from river banks and steep coasts. Shifted sand and mud horizontally on beaches and flat land. Changed level of water in wells. Threw water on banks of canals, 1akes, rivers, etc. Damage serious to dams, dikes, embankments. Severe to well-built wooden structures and bridges, some destroyed. Developed dangerous cracks in excellent brick walls. Destroyed most masonry and frame structures, also their foundations. Bent railroad rails slightly. Tore apart, or crushed endwise, pipe lines buried in earth. Open cracks and broad wavy folds in cement pavements and asphalt road surfaces.

$X I$. Disturbances in ground many and widespread, varying with ground material. Broad fissures, earth slumps, and land slips in soft, wet ground. Ejected water in large amounts charged with sand and mud. Caused sea-waves ("tidal" waves) of significant magnitude. Damage severe to wood-frame structures, especially near shock centers. Great to dams, dikes, embankments often for long distances. Few, if any (masonry) structures remained standing. Destroyed large well-built bridges by the wrecking of supporting piers, or pillars. Affected yielding wooden bridges less. Bent railroad rails greatly, and thrust them endwise. Put pipe lines buried in earth completely out of service.

XII. Damage tota1--practically all works of construction damaged greatly or destroyed. Disturbances in ground great and varied, numerous shearing cracks. Landslides, falls of rock of significant character, slumping of river banks, etc., numerous and extensive. Wrenched loose, tore off, large rock masses. Fault slips in firm rock, with notable horizontal and vertical offset displacements. Water channels, surface and underground, disturbed and modified greatly. Dammed lakes, produced waterfalls, deflected rivers, etc. Waves seen on ground surfaces (actually seen, probably, in some cases). Distorted lines of sight and level. Threw objects upward in to the air. 
Table 1.--Summary of U.S. earthquakes for January-March 1978

[Sources of the hypocenters and magnitudes: (A) Geophysical Institute, University of Alaska, Fairbanks; (B) University of California, Berkeley; (D) University of Montana, Missoula; (E) U.S. Department of Energy, Las Vegas, Nevada; (G) U.S. Geological Survey, National Earthquake Information Service; (H) U.S. Geological Survey, Hawaiian Volcano Observatory; (J) Weston Observatory, Massachusetts; (L) Lamont-Doherty Geological Observatory, Palisades, N.Y.; (M) National Oceanic and Atmospheric Administration, Alaska Tsunami Warning Center, Palmer; (P) California Institute of Technology, Pasadena; (S) St. Louis University, St. Louis, Missouri; (T) University of Oklahoma, Leonard; (U) University of Utah, Salt Lake City; (V) Virginia Polytechnic Institute and State University, Blacksburg; (W) University of Washington, Seattle. N, Normal depth; UTC, Universal Coordinated Tíme. For names of local time zones, see figures 2 and 3 . Leaders (...) indicate no information available]

Date
$\begin{aligned} & (1978) \\ & \text { (UTC) }\end{aligned}$

ALABAMA

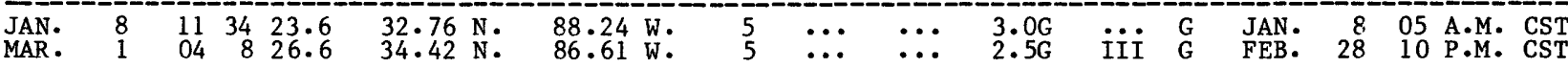

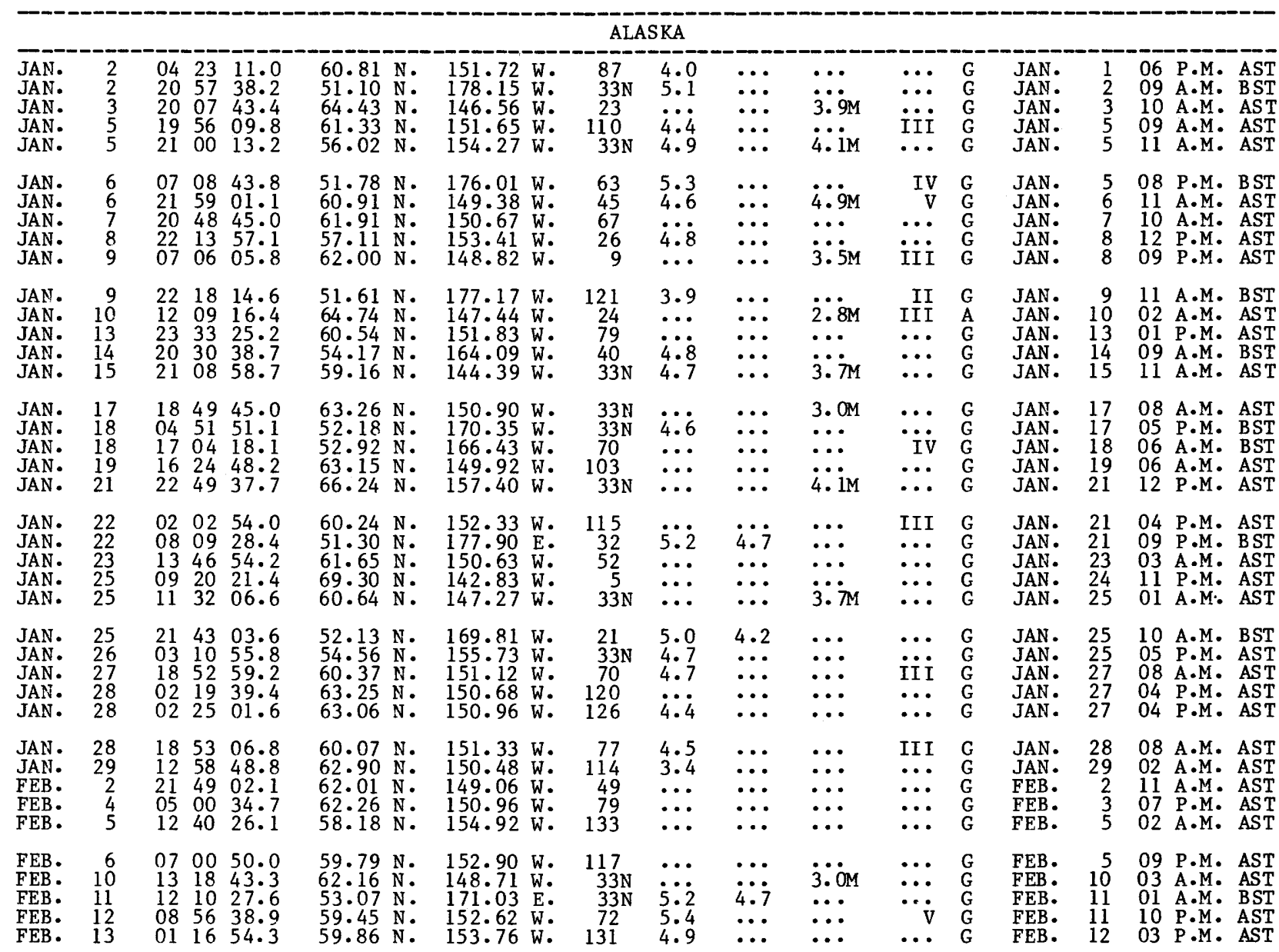


Table 1.--Summary of U.S. earthquakes for January-March 1978--Continued

Date
$\begin{gathered}1978) \\ \text { Origin time }\end{gathered}$

CALIFORNIA-Con $t$ in ued

\begin{tabular}{|c|c|c|c|c|c|c|c|c|c|c|c|c|c|c|}
\hline $\begin{array}{l}\text { AN. } \\
\text { AN: } \\
\text { AN. } \\
\text { AN. }\end{array}$ & $\begin{array}{l}24 \\
24 \\
25 \\
28 \\
29\end{array}$ & $\begin{array}{lll}08 & 13 & 21.7 \\
12 & 26 & 44.2 \\
10 & 40 & 50.0 \\
08 & 49 & 02.5 \\
01 & 55 & 49.8\end{array}$ & $\begin{array}{l}33.27 \mathrm{~N} \cdot \\
34.68 \mathrm{~N}: \\
34.32 \mathrm{~N}: \\
39.40 \mathrm{~N}: \\
37.18 \mathrm{~N} .\end{array}$ & $\begin{array}{l}116.00 \mathrm{~W} . \\
118.93 \mathrm{~W} . \\
118.33 \mathrm{~W} . \\
123.35 \mathrm{~W} . \\
117.85 \mathrm{~W} .\end{array}$ & $\begin{array}{r}5 \\
5 \\
5 \\
5 \\
11\end{array}$ & $\begin{array}{l}\cdots \\
\cdots \\
\cdots\end{array}$ & $\begin{array}{l}\cdots \\
\because \cdots \\
\because \cdots\end{array}$ & $\begin{array}{l}3.3 \mathrm{P} \\
3.0 \mathrm{P} \\
2.7 \mathrm{P} \\
2.5 \mathrm{~B} \\
2.9 \mathrm{~B}\end{array}$ & 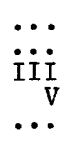 & & $\begin{array}{l}\text { JAN. } \\
\text { JAN. } \\
\text { JAN. } \\
\text { JAN. } \\
\text { JAN. }\end{array}$ & $\begin{array}{l}24 \\
24 \\
25 \\
28 \\
28\end{array}$ & $\begin{array}{l}04 \\
02 \\
00 \\
05\end{array}$ & $\begin{array}{l}\text { A.M. PST } \\
\text { A.M. PST } \\
\text { A.M. PST } \\
\text { A.M. PST } \\
\text { P.M. PST }\end{array}$ \\
\hline $\begin{array}{l}\text { IAN. } \\
\text { IAN: } \\
\text { EB: } \\
\text { EB: }\end{array}$ & $\begin{array}{r}29 \\
31 \\
2 \\
5 \\
6\end{array}$ & $\begin{array}{rrr}02 & 56 & 9 \cdot 0 \\
14 & 18 & 10.0 \\
14 & 8 & 44.6 \\
09 & 53 & 41.5 \\
00 & 39 & 25.8\end{array}$ & $\begin{array}{l}34.03 \mathrm{~N} \cdot \\
34.18 \mathrm{~N} . \\
32.97 \\
34.32 \\
34.03 \\
\mathrm{~N}\end{array}$ & $\begin{array}{l}115.62 \mathrm{~W} . \\
118.63 \mathrm{~W} . \\
115.85 \mathrm{~W} . \\
116.72 \mathrm{~W} . \\
116.78 \mathrm{~W} .\end{array}$ & $\begin{array}{l}4 \\
6 \\
2 \\
5\end{array}$ & $\begin{array}{l}\ldots \\
\cdots \\
\cdots \\
\cdots\end{array}$ & $\begin{array}{l}\cdots \\
\because \cdots\end{array}$ & $\begin{array}{l}2.1 \mathrm{P} \\
3.0 \mathrm{P} \\
3.6 \mathrm{P} \\
3.1 \mathrm{P}\end{array}$ & $\begin{array}{l}\ddot{i} \dot{I} \\
\ddot{I} \ddot{I} \\
\dot{I}\end{array}$ & & $\begin{array}{l}\text { JAN. } \\
\text { JAN. } \\
\text { FEB. } \\
\text { FEB. } \\
\text { FEB. }\end{array}$ & $\begin{array}{r}28 \\
31 \\
2 \\
5 \\
5\end{array}$ & $\begin{array}{l}06 \\
01 \\
04\end{array}$ & $\begin{array}{l}\text { P.M. PST } \\
\text { A.M. PST } \\
\text { A.M. PST } \\
\text { A.M. PST } \\
\text { P.M. PST }\end{array}$ \\
\hline $\begin{array}{l}\mathrm{EB} \\
\mathrm{EB} \\
\mathrm{EB} \\
\mathrm{EB} \\
\mathrm{EB} \\
\mathrm{EB}\end{array}$ & $\begin{array}{r}6 \\
6 \\
7 \\
8 \\
11\end{array}$ & $\begin{array}{rrr}01 & 1 & 28 \cdot 9 \\
12 & 57 & 14 \cdot 2 \\
13 & 39 & 22.7 \\
20 & 8 & 21.2 \\
01 & 45 & 30.5\end{array}$ & 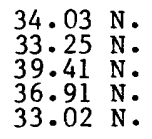 & $\begin{array}{l}116.78 \mathrm{~W} . \\
115.57 \mathrm{~W} . \\
123.17 \mathrm{~W} . \\
121.70 \mathrm{~W} . \\
115.53 \mathrm{~W} .\end{array}$ & $\begin{array}{r}6 \\
4 \\
5 \\
14 \\
7\end{array}$ & $\begin{array}{l}\cdots \\
\cdots \\
\cdots\end{array}$ & .. & $\begin{array}{l}3.3 \mathrm{P} \\
3.4 \mathrm{P} \\
2.7 \mathrm{~B} \\
3.0 \mathrm{~B} \\
2.5 \mathrm{P}\end{array}$ & $\begin{array}{r}\text { III } \\
\mathrm{V} \\
\mathrm{V} \\
\ddot{\text { II }}\end{array}$ & & $\begin{array}{l}\text { FEB. } \\
\text { FEB. } \\
\text { FEB. } \\
\text { FEB. } \\
\text { FEB. }\end{array}$ & $\begin{array}{r}5 \\
6 \\
7 \\
8 \\
10\end{array}$ & $\begin{array}{l}04 \\
05 \\
12 \\
05\end{array}$ & $\begin{array}{l}\text { P.M. PST } \\
\text { A.M. PST } \\
\text { A.M. PST } \\
\text { P.M. PST } \\
\text { P.M. PST }\end{array}$ \\
\hline $\begin{array}{l}\text { FEB. } \\
\text { FEB: } \\
\text { FEB: } \\
\text { FEB: } \\
\text { FEB. }\end{array}$ & $\begin{array}{l}11 \\
11 \\
13 \\
13 \\
14\end{array}$ & $\begin{array}{rrr}01 & 45 & 51.0 \\
02 & 25 & 51.4 \\
06 & 8 & 46.5 \\
18 & 4 & 6.3 \\
16 & 33 & 46.9\end{array}$ & $\begin{array}{l}33.02 \mathrm{~N} . \\
33.02 \\
40.23 \\
34.02 \\
38.41 \\
\mathrm{~N} .\end{array}$ & $\begin{array}{l}115.53 \mathrm{~W} . \\
115.53 \mathrm{~W} . \\
122.11 \mathrm{~W} . \\
117.22 \mathrm{~W} . \\
122.65 \mathrm{~W} .\end{array}$ & $\begin{array}{r}5 \\
6 \\
15 \\
6 \\
8\end{array}$ & $\begin{array}{l}\cdots \\
\cdots \\
\cdots\end{array}$ & $\begin{array}{l}\cdots \\
\because \cdots \\
\therefore\end{array}$ & $\begin{array}{l}2.5 \mathrm{P} \\
3.1 \mathrm{P} \\
3.3 \mathrm{~B} \\
2.9 \mathrm{P} \\
2.8 \mathrm{~B}\end{array}$ & $\begin{array}{l}\text { II } \\
\text { III } \\
\dddot{\text { II }} \\
\text { II }\end{array}$ & & $\begin{array}{l}\text { FEB. } \\
\text { FEB. } \\
\text { FEB. } \\
\text { FEB. } \\
\text { FEB. }\end{array}$ & $\begin{array}{l}10 \\
10 \\
12 \\
13 \\
14\end{array}$ & $\begin{array}{l}06 \\
10 \\
10 \\
08\end{array}$ & $\begin{array}{l}\text { P.M. } \\
\text { P.M: } \\
\text { P.M. } \\
\text { A.M: } \\
\text { A.M. }\end{array}$ \\
\hline $\begin{array}{l}\text { FEB. } \\
\text { FEB: } \\
\text { FEB; } \\
\text { FEB: } \\
\text { FEB. }\end{array}$ & $\begin{array}{l}14 \\
14 \\
16 \\
17 \\
17\end{array}$ & $\begin{array}{rrr}20 & 3 & 9.5 \\
21 & 3 & 55.4 \\
10 & 54 & 35.3 \\
07 & 35 & 26.7 \\
10 & 13 & 45.2\end{array}$ & $\begin{array}{l}38.42 \mathrm{~N} . \\
40.30 \mathrm{~N}: \\
33.42 \mathrm{~N}: \\
40.10 \mathrm{~N} . \\
35.72 \mathrm{~N} .\end{array}$ & $\begin{array}{l}122.66 \mathrm{~W} . \\
124.27 \mathrm{~W} . \\
116.40 \mathrm{~W} . \\
121.43 \mathrm{~W} . \\
115.92 \mathrm{~W} .\end{array}$ & $\begin{array}{r}9 \\
10 \\
5 \\
10 \\
4\end{array}$ & $\begin{array}{l}\cdots \\
\cdots \\
\cdots\end{array}$ & $\begin{array}{l}\cdots \\
\because \cdots \\
\cdots\end{array}$ & $\begin{array}{l}2.5 \mathrm{~B} \\
4 . \mathrm{OB} \\
3.0 \mathrm{P} \\
3.0 \mathrm{~B} \\
3.0 \mathrm{P}\end{array}$ & $\begin{array}{l}\text { II } \\
\text { IV } \\
\cdots \\
\cdots \\
\cdots\end{array}$ & & $\begin{array}{l}\text { FEB. } \\
\text { FEB. } \\
\text { FEB. } \\
\text { FEB. } \\
\text { FEB. }\end{array}$ & $\begin{array}{l}14 \\
14 \\
16 \\
16 \\
17\end{array}$ & $\begin{array}{l}12 \\
01 \\
02 \\
11 \\
02\end{array}$ & $\begin{array}{ll}P . M . M & \text { PST } \\
\text { P.M. } & \text { PST } \\
A \cdot M . & \text { PST } \\
\text { P.M. } & \text { PST } \\
A . M . & \text { PST }\end{array}$ \\
\hline $\begin{array}{l}\text { FEB } \\
\text { FEB. } \\
\text { FEB: } \\
\text { FEB: } \\
\text { MAR. }\end{array}$ & $\begin{array}{r}20 \\
23 \\
23 \\
28 \\
1\end{array}$ & $\begin{array}{rrr}23 & 38 & 54.3 \\
10 & 14 & 6.1 \\
16 & 43 & 3.7 \\
16 & 20 & 52.1 \\
04 & 54 & 31.2\end{array}$ & $\begin{array}{l}35.65 \mathrm{~N} . \\
38.29 \mathrm{~N} . \\
32.82 \mathrm{~N}: \\
40.51 \mathrm{~N} \\
34.53 \mathrm{~N} .\end{array}$ & $\begin{array}{l}121.04 \mathrm{~W} . \\
119.11 \mathrm{~W} . \\
115.600 \mathrm{~W} . \\
121.93 \mathrm{~W} . \\
116.77 \text { W. }\end{array}$ & $\begin{array}{r}5 \\
7 \\
19 \\
5 \\
5\end{array}$ & & & $\begin{array}{l}3.1 \mathrm{~B} \\
3.2 \mathrm{~B} \\
3.6 \mathrm{P} \\
3.0 \mathrm{~B} \\
4.4 \mathrm{P}\end{array}$ & ... & B & $\begin{array}{l}\text { FEB. } \\
\text { FEB. } \\
\text { FEB. } \\
\text { FEB. } \\
\text { FEB. }\end{array}$ & $\begin{array}{l}20 \\
23 \\
23 \\
28 \\
28\end{array}$ & $\begin{array}{l}03 \\
02 \\
08 \\
08 \\
08\end{array}$ & $\begin{array}{l}A \cdot M \cdot \\
A \cdot M \cdot \\
A \cdot M \bullet \\
P \cdot M\end{array}$ \\
\hline $\begin{array}{l}\text { R. } \\
\text { R: } \\
\text { R: } \\
\text { R: }\end{array}$ & $\begin{array}{r}3 \\
7 \\
8 \\
13 \\
14\end{array}$ & $\begin{array}{rrr}18 & 37 & 24.0 \\
15 & 28 & 33.7 \\
14 & 49 & 34.9 \\
16 & 38 & 15.7 \\
16 & 9 & 51.2\end{array}$ & $\begin{array}{l}36.02 \mathrm{~N} . \\
36.00 \mathrm{~N} \\
33.83 \\
33.93 \\
33.95 \\
\mathrm{~N} .\end{array}$ & $\begin{array}{l}117.63 \mathrm{~W} . \\
117.63 \mathrm{~W} . \\
117.88 \mathrm{~W} . \\
117.98 \mathrm{~W} . \\
117.97 \mathrm{~W} .\end{array}$ & $\begin{array}{l}3 \\
5 \\
5\end{array}$ & & & $\begin{array}{l}3.0 \mathrm{P} \\
3.3 \mathrm{P} \\
2.9 \mathrm{P} \\
3.2 \mathrm{P} \\
2.5 \mathrm{P}\end{array}$ & \begin{tabular}{l}
$\because \cdots$ \\
\hdashline$\ddot{I V}$ \\
VI \\
IV
\end{tabular} & & $\begin{array}{l}\text { MAR. } \\
\text { MAR: } \\
\text { MAR: } \\
\text { MAR. } \\
\text { MAR. }\end{array}$ & $\begin{array}{r}3 \\
7 \\
8 \\
13 \\
14\end{array}$ & $\begin{array}{l}06 \\
08 \\
08\end{array}$ & $\begin{array}{l}\text { A.M. } \\
\text { A.M. } \\
\text { A.M: }\end{array}$ \\
\hline $\begin{array}{l}\mathrm{AR} \text {. } \\
\mathrm{AR} \text {. } \\
\mathrm{AR} \text {. }\end{array}$ & $\begin{array}{l}14 \\
15 \\
15 \\
18 \\
20\end{array}$ & $\begin{array}{lrr}23 & 59 & 55.0 \\
04 & 2 & 49.4 \\
04 & 9 & 39.7 \\
08 & 20 & 36.5 \\
00 & 7 & 56.7\end{array}$ & $\begin{array}{l}34.00 \mathrm{~N} . \\
32.78 \mathrm{~N} . \\
32.78 \mathrm{~N} . \\
35.32 \mathrm{~N} \\
36.00 \mathrm{~N} .\end{array}$ & $\begin{array}{l}118.68 \mathrm{~W} . \\
115.53 \mathrm{~W} . \\
115.57 \mathrm{~W} . \\
118.52 \mathrm{~W} . \\
117.87 \mathrm{~W} .\end{array}$ & $\begin{array}{r}12 \\
5 \\
5 \\
5 \\
1\end{array}$ & & & $\begin{array}{l}3.2 \mathrm{P} \\
2.9 \mathrm{P} \\
3.2 \mathrm{P} \\
3.5 \mathrm{P} \\
3.0 \mathrm{P}\end{array}$ & & $\mathrm{P}$ & $\begin{array}{l}\text { MAR. } \\
\text { MAR : } \\
\text { MAR . } \\
\text { MAR . } \\
\text { MAR. }\end{array}$ & $\begin{array}{l}14 \\
14 \\
14 \\
18 \\
19\end{array}$ & $\begin{array}{l}08 \\
00 \\
04\end{array}$ & $\begin{array}{l}\text { P.M. } \\
\text { P.M: } \\
\text { P.M. } \\
\text { A.M: } \\
\text { P.M. }\end{array}$ \\
\hline $\begin{array}{l}\text { AR: } \\
\text { AR: } \\
\text { AR: } \\
\text { AR: }\end{array}$ & $\begin{array}{l}21 \\
21 \\
22 \\
22 \\
26\end{array}$ & $\begin{array}{rrr}14 & 5 & 12.1 \\
20 & 17 & 6.8 \\
06 & 33 & 17.3 \\
08 & 40 & 35.3 \\
00 & 27 & 4.8\end{array}$ & $\begin{array}{l}40.44 \mathrm{~N} . \\
40.44 \mathrm{~N} . \\
40.12 \mathrm{~N}: \\
40.09 \mathrm{~N} . \\
39.21 \mathrm{~N} .\end{array}$ & $\begin{array}{l}124.45 \mathrm{~W} . \\
124.46 \mathrm{~W} . \\
122.95 \mathrm{~W} . \\
122.95 \mathrm{~W} . \\
123.17 \mathrm{~W} .\end{array}$ & $\begin{array}{r}11 \\
13 \\
5 \\
14 \\
9\end{array}$ & $\ddot{4} \cdot \dot{9}$ & & $\begin{array}{l}3.5 \mathrm{~B} \\
3.4 \mathrm{~B} \\
3.2 \mathrm{~B} \\
3.2 \mathrm{~B} \\
4.4 \mathrm{~B}\end{array}$ & $\begin{array}{l}\text { IV } \\
\because \because \\
\because \because \\
\mathrm{VI}\end{array}$ & $\begin{array}{l}\text { B } \\
\text { B } \\
\text { B }\end{array}$ & $\begin{array}{l}\text { MAR. } \\
\text { MAR. } \\
\text { MAR. } \\
\text { MAR. } \\
\text { MAR. }\end{array}$ & $\begin{array}{l}21 \\
21 \\
21 \\
22 \\
25\end{array}$ & $\begin{array}{l}12 \\
10 \\
00 \\
04\end{array}$ & $\begin{array}{l}\text { A.M. PST } \\
\text { P.M. PST } \\
\text { P.M. PST } \\
\text { A.M. PST } \\
\text { P.M. PST }\end{array}$ \\
\hline $\begin{array}{l}\text { MAR: } \\
\text { MAR: } \\
\text { MAR: } \\
\text { MAR: } \\
\text { MAR: }\end{array}$ & $\begin{array}{l}26 \\
26 \\
26 \\
26\end{array}$ & $\begin{array}{lll}00 & 34 & 11.9 \\
01 & 19 & 10.0 \\
02 & 29 & 16.9 \\
04 & 28 & 18.6 \\
10 & 56 & 22.4\end{array}$ & $\begin{array}{l}39.21 \mathrm{~N} . \\
39.20 \mathrm{~N} \\
39.21 \\
39.23 \\
36.93 \\
\mathrm{~N} .\end{array}$ & $\begin{array}{l}123.14 \mathrm{~W} . \\
123.17 \mathrm{~W} . \\
123.17 \mathrm{~W} . \\
123.19 \mathrm{~W} . \\
117.46 \mathrm{~W} .\end{array}$ & $\begin{array}{r}5 \\
10 \\
5 \\
3\end{array}$ & & & $\begin{array}{l}3 \cdot 1 \mathrm{~B} \\
3 \cdot 6 \mathrm{~B} \\
3 \cdot 3 \mathrm{~B} \\
3 \cdot 5 \mathrm{~B} \\
3.2 \mathrm{~B}\end{array}$ & $\begin{array}{l}\text { IV } \\
\text { IV } \\
\text { IV } \\
\text { IV }\end{array}$ & $\begin{array}{l}\text { B } \\
\text { B } \\
\text { B } \\
\text { B } \\
\text { B }\end{array}$ & $\begin{array}{l}\text { MAR. } \\
\text { MAR: } \\
\text { MAR: } \\
\text { MAR: } \\
\text { MAR. }\end{array}$ & $\begin{array}{l}25 \\
25 \\
25 \\
25 \\
26\end{array}$ & $\begin{array}{l}0 \\
0 \\
0 \\
0\end{array}$ & $\begin{array}{l}\text { P.M: } \\
\text { P.M: } \\
\text { A.M. }\end{array}$ \\
\hline $\begin{array}{l}\text { MAR: } \\
\text { MAR: } \\
\text { MAR: } \\
\text { MAR: } \\
\text { MAR: }\end{array}$ & $\begin{array}{l}27 \\
27 \\
27 \\
27\end{array}$ & $\begin{array}{rrr}04 & 27 & 1.1 \\
04 & 29 & 13.9 \\
08 & 44 & 3.0 \\
16 & 15 & 33.6 \\
01 & 3 & 27.7\end{array}$ & $\begin{array}{l}34.85 \mathrm{~N} . \\
37.00 \mathrm{~N}: \\
40.43 \mathrm{~N}: \\
39.19 \mathrm{~N}: \\
38.48 \mathrm{~N} .\end{array}$ & $\begin{array}{l}117.08 \mathrm{~W} . \\
117.83 \mathrm{~W} . \\
124.31 \mathrm{~W} . \\
123.16 \mathrm{~W} . \\
123.34 \mathrm{~W} .\end{array}$ & $\begin{array}{r}5 \\
2 \\
14 \\
8 \\
7\end{array}$ & & & $\begin{array}{l}3.1 \mathrm{P} \\
3.1 \mathrm{~B} \\
3.2 \mathrm{~B} \\
3.0 \mathrm{~B} \\
3.6 \mathrm{~B}\end{array}$ & $\because \ddot{v}$ & B & $\begin{array}{l}\text { MAR. } \\
\text { MAR . } \\
\text { MAR. } \\
\text { MAR. } \\
\text { MAR. }\end{array}$ & $\begin{array}{l}26 \\
26 \\
27 \\
27 \\
30\end{array}$ & $\begin{array}{l}0 \\
0 \\
0\end{array}$ & $\begin{array}{l}\text { A.M: } \\
\text { A.M. } \\
\text { P.M. }\end{array}$ \\
\hline
\end{tabular}


Table 1.--Summary of U.S. earthquakes for January-March 1978--Continued

$\begin{array}{lllll}\text { Date } & \begin{array}{l}\text { Origin time } \\ (1978)\end{array} & \text { LatC) } & \text { Lop } & \begin{array}{l}\text { Depth } \\ (\mathrm{km})\end{array}\end{array}$

CALIFORNIA-OFF THE COAST

\begin{tabular}{|c|c|c|c|c|c|c|c|c|c|c|c|c|c|c|c|}
\hline $\begin{array}{l}\text { JAN. } \\
\text { JAN. } \\
\text { JAN. } \\
\text { JAN. } \\
\text { JAN. }\end{array}$ & $\begin{array}{l}2 \\
11 \\
12 \\
18 \\
24\end{array}$ & $\begin{array}{lr}06 & 39 \\
18 & 25 \\
08 & 53 \\
16 & 8 \\
13 & 2\end{array}$ & $\begin{array}{r}19.5 \\
34.3 \\
50.5 \\
39.2 \\
3.9\end{array}$ & $\begin{array}{l}40.86 \\
40.60 \\
40.90 \\
40.53 \\
34.61\end{array}$ & $\begin{array}{l}\text { N. } \\
\text { N. } \\
\text { N. } \\
\text { N. }\end{array}$ & $\begin{array}{l}125.23 \mathrm{~W} . \\
126.20 \mathrm{~W} . \\
125.75 \\
124.83 \\
121.62 \\
\text { W. }\end{array}$ & $\begin{array}{r}16 \\
5 \\
5 \\
25 \\
10\end{array}$ & $\begin{array}{l}3.9 \\
\cdots \\
\cdots \\
\cdots\end{array}$ & $\begin{array}{l}4.1 \\
\cdots \\
\cdots \\
\cdots\end{array}$ & $\begin{array}{l}4 \cdot 1 \mathrm{~B} \\
3 \cdot 8 \mathrm{~B} \\
3 \cdot 0 \mathrm{~B} \\
3.7 \mathrm{~B} \\
3.0 \mathrm{~B}\end{array}$ & $\begin{array}{l}\cdots \\
\cdots \\
\cdots\end{array}$ & $\begin{array}{l}\text { B } \\
\text { B } \\
\text { B } \\
\text { B } \\
\text { G }\end{array}$ & $\begin{array}{l}\text { JAN. } \\
\text { JAN. } \\
\text { JAN. } \\
\text { JAN. } \\
\text { JAN. }\end{array}$ & $\begin{array}{l}1 \\
11 \\
12 \\
18 \\
24\end{array}$ & $\begin{array}{l}10 \text { P.M. } \\
10 \text { A.M. } \\
00 \text { A.M. } \\
08 \text { A.M. } \\
05 \text { A.M. }\end{array}$ \\
\hline
\end{tabular}

FLOR IDA

JAN. 12 21 10 NEAR HAINES CITY

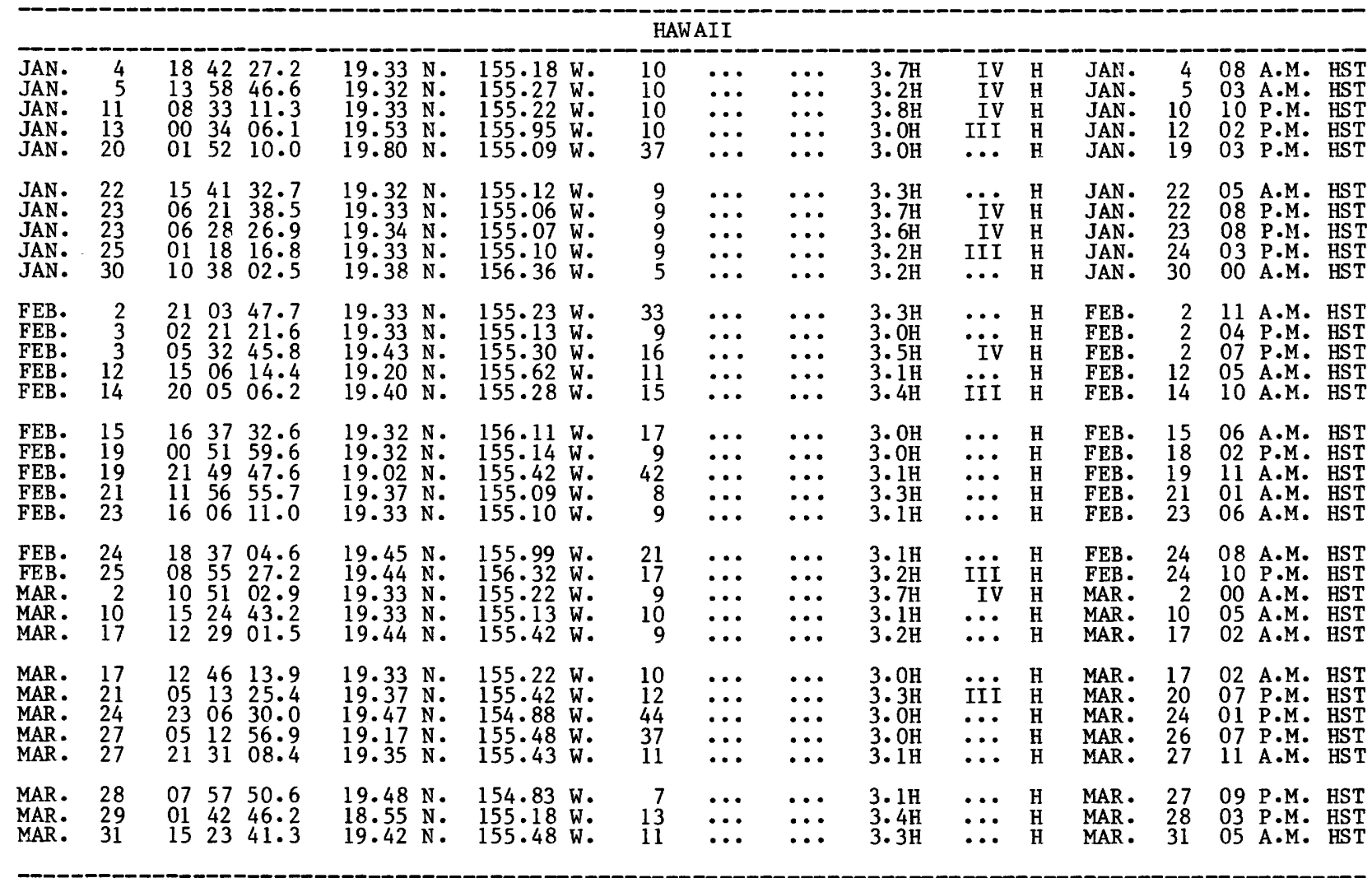

IDAHO

\begin{tabular}{|c|c|c|c|c|c|c|c|c|c|c|c|c|c|}
\hline $\begin{array}{l}\text { EB. } \\
\text { EB. } \\
\text { EB. }\end{array}$ & $\begin{array}{l}13 \\
22 \\
25 \\
19 \\
22\end{array}$ & $\begin{array}{lll}17 & 35 & 36.9 \\
00 & 37 & 1.1 \\
21 & 22 & 21.2 \\
02 & 33 & 48.7 \\
14 & 30 & 17.0\end{array}$ & $\begin{array}{l}45.06 \mathrm{~N} . \\
44.61 \mathrm{~N} . \\
44.64 \mathrm{~N} . \\
44.50 \mathrm{~N} . \\
44.28 \mathrm{~N} .\end{array}$ & $\begin{array}{l}114.44 \mathrm{~W} . \\
115.06 \mathrm{~W} \\
113.80 \mathrm{~W} . \\
114.33 \mathrm{~W} . \\
115.47 \mathrm{~W} .\end{array}$ & $\begin{array}{l}5 \\
5 \\
5\end{array}$ & $\begin{array}{l}\ddot{3} \ddot{9} \\
\ddot{4} \\
\ddot{4} \ddot{5}\end{array}$ & $\begin{array}{l}\cdots \\
\cdots \\
\cdots\end{array}$ & $\begin{array}{l}3.3 \mathrm{G} \\
3.8 \mathrm{G} \\
3.3 \mathrm{G} \\
4.1 \mathrm{G}\end{array}$ & $\begin{array}{l}\cdots \\
\cdots \\
\cdots \\
\cdots\end{array}$ & $\begin{array}{l}\mathrm{G} \\
\mathrm{G} \\
\mathrm{G} \\
\mathrm{G}\end{array}$ & $\begin{array}{l}\text { FEB. } \\
\text { FEB. } \\
\text { FEB. } \\
\text { MAR. } \\
\text { MAR. }\end{array}$ & $\begin{array}{l}13 \\
21 \\
25 \\
18 \\
22\end{array}$ & $\begin{array}{ll}10 & \mathrm{~A} \\
05 & \mathrm{P} \\
02 & \mathrm{P} \\
07 & \mathrm{P}\end{array}$ \\
\hline
\end{tabular}

MAINE

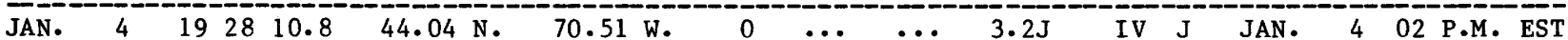


Table 1.--Summary of U.S. earthquakes for January-March 1978--Continued

$\begin{array}{llll}\text { Date } & \text { Origin time } & \text { Lather } & \begin{array}{l}\text { Depth } \\ (\mathbf{k m})\end{array}\end{array}$

MONTANA

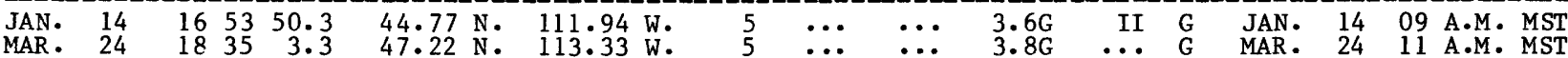

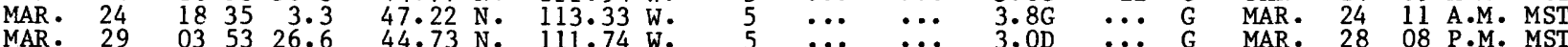

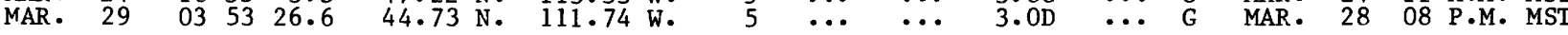

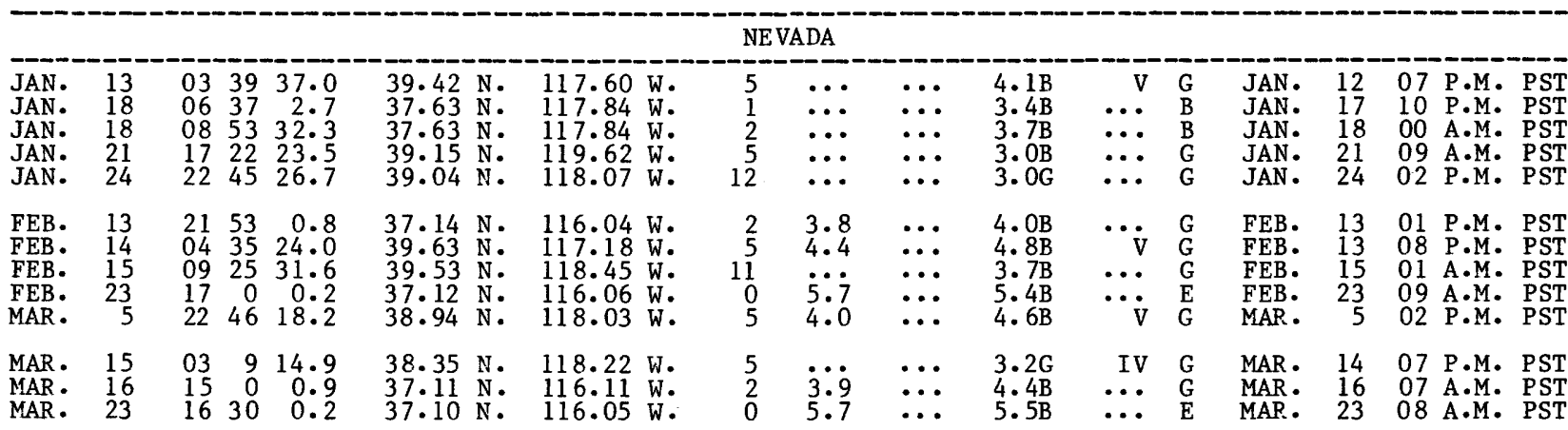

NEW HAMPSHIRE

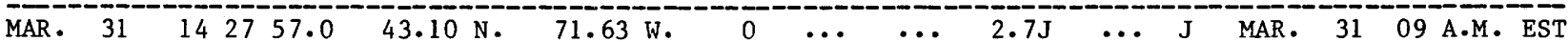
NEW YORK

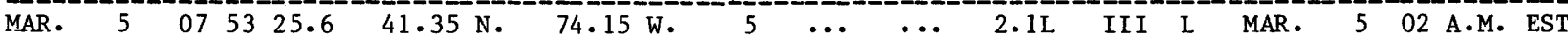

$$
\text { NORTH CAROLINA }
$$

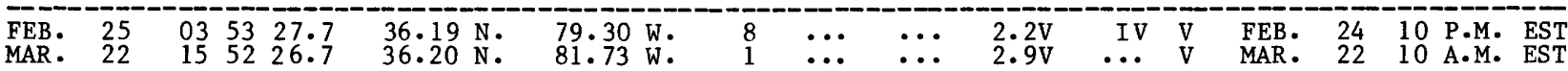

$\overline{\mathrm{MAR}} \mathrm{9}$

\section{OREGON-OFF THE COAST}

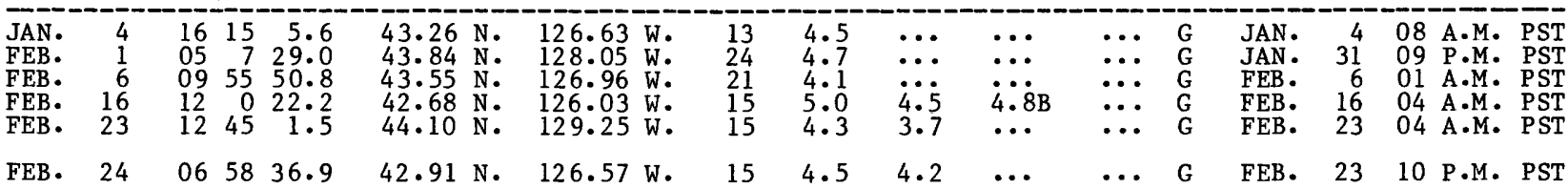

SOUTH CAROL INA

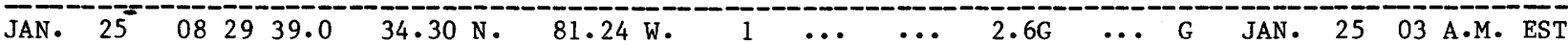
TENNESSEE

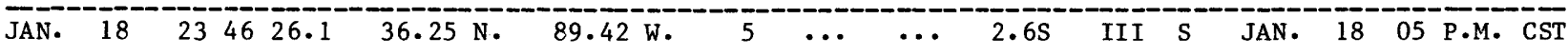

\section{TEXAS}

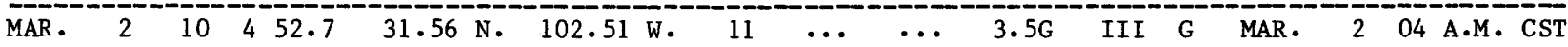

\section{UTAH}

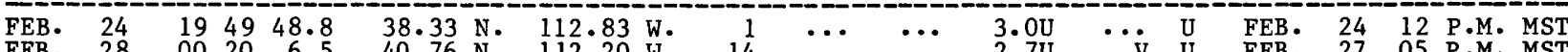

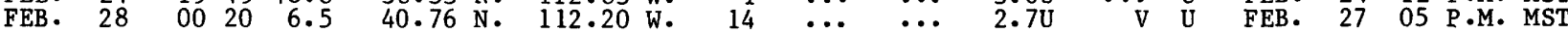


Table 1.--Summary of U.S. earthquakes for January-March 1978--Continued

Date
$(1978)$$\quad \begin{aligned} & \text { Origin time } \\ & \text { (UTC) }\end{aligned}$

UTAH--Continued

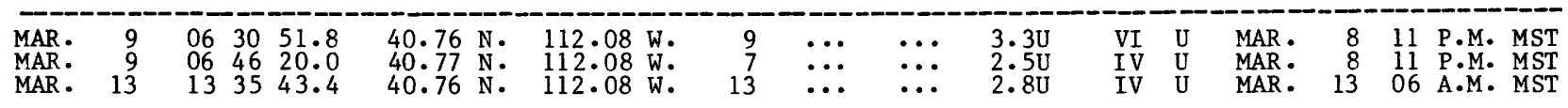

VIRGINIA

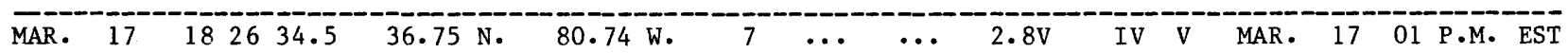

\begin{tabular}{|c|c|c|c|c|c|c|c|c|c|c|c|c|c|}
\hline \multicolumn{14}{|c|}{ WASHINGTON } \\
\hline $\begin{array}{l}\text { MAR. } \\
\text { MAR } \\
\text { MAR: } \\
\text { MAR. }\end{array}$ & $\begin{array}{r}5 \\
11 \\
29 \\
31\end{array}$ & $\begin{array}{rrr}18 & 13 & 35.9 \\
15 & 52 & 11.2 \\
12 & 16 & 38.4 \\
08 & 3 & 0.2\end{array}$ & $\begin{array}{l}48.06 \mathrm{~N} . \\
47.42 \mathrm{~N} . \\
48.20 \mathrm{~N} \\
47.42 \mathrm{~N} .\end{array}$ & $\begin{array}{l}123.00 \mathrm{~W} . \\
122.71 \mathrm{~W} . \\
122.76 \mathrm{~W} . \\
122.72 \mathrm{~W} .\end{array}$ & $\begin{array}{l}57 \\
25 \\
24 \\
23\end{array}$ & $\begin{array}{l}4.0 \\
\ldots \\
\ldots\end{array}$ & $\because \ldots$ & $\begin{array}{l}3.3 \mathrm{G} \\
4.8 \mathrm{G} \\
2.7 \mathrm{G} \\
4.2 \mathrm{G}\end{array}$ & $\begin{array}{l}\text { IV } \\
\text { VI } \\
\text { IV } \\
\text { V }\end{array}$ & $\begin{array}{l}W \\
W \\
W \\
W\end{array}$ & $\begin{array}{l}\text { MAR . } \\
\text { MAR . } \\
\text { MAR. } \\
\text { MAR . }\end{array}$ & $\begin{array}{l}5 \\
11 \\
29 \\
31\end{array}$ & $\begin{array}{ll}10 & \text { A.M. PST } \\
07 & \text { A.M. PST } \\
04 & \text { A.M. PST } \\
00 & \text { A.M. PST }\end{array}$ \\
\hline
\end{tabular}

\begin{tabular}{|c|c|c|c|c|c|c|c|c|c|c|c|c|c|c|c|c|}
\hline \multicolumn{17}{|c|}{ WYOMING } \\
\hline $\begin{array}{l}\text { JAN. } \\
\text { FEB. } \\
\text { FEB. } \\
\text { FEB. } \\
\text { MAR. }\end{array}$ & $\begin{array}{r}16 \\
2 \\
2 \\
7 \\
7\end{array}$ & $\begin{array}{l}03 \\
00 \\
12 \\
05 \\
01\end{array}$ & $\begin{array}{l}50 \\
36 \\
35 \\
3 \\
10\end{array}$ & $\begin{array}{l}3.1 \\
25.6 \\
56.2 \\
10.4 \\
47.6\end{array}$ & $\begin{array}{l}42.43 \\
44.39 \\
44.38 \\
42.50 \\
44.43\end{array}$ & $\begin{array}{l}\text { N: } \\
\text { N: } \\
\text { N: } \\
\text { N: } \\
\text { N. }\end{array}$ & $\begin{array}{l}105.31 \mathrm{~W} . \\
110.81 \mathrm{~W} . \\
110.83 \mathrm{~W} . \\
109.70 \mathrm{~W} . \\
110.84 \mathrm{~W} .\end{array}$ & $\begin{array}{r}5 \\
5 \\
4 \\
30 \\
5\end{array}$ & $\begin{array}{l}\ddot{3} \dot{6} \\
\ddot{0} \\
\ddot{3} . \overline{7}\end{array}$ & $\begin{array}{l}\cdots \\
\cdots \\
\cdots \\
\cdots\end{array}$ & $\begin{array}{l}3.0 \mathrm{G} \\
3.4 \mathrm{G} \\
3.1 \mathrm{G} \\
3.3 \mathrm{G} \\
3.5 \mathrm{G}\end{array}$ & $\begin{array}{l}\text { III } \\
\text { III } \\
\text { III } \\
\cdots \dot{v}\end{array}$ & $\begin{array}{l}G \\
G \\
G \\
G \\
G\end{array}$ & $\begin{array}{l}\text { JAN. } \\
\text { FEB. } \\
\text { FEB. } \\
\text { FEB. } \\
\text { MAR. }\end{array}$ & $\begin{array}{r}15 \\
1 \\
2 \\
6 \\
6\end{array}$ & $\begin{array}{ll}08 & \text { P.M. MST } \\
05 & \text { P.M. MST } \\
05 & \text { A.M. MST } \\
10 & \text { P.M. MST } \\
06 & \text { P.M. MST }\end{array}$ \\
\hline $\begin{array}{l}\text { MAR } \\
\text { MAR . }\end{array}$ & $\begin{array}{r}7 \\
10\end{array}$ & $\begin{array}{l}07 \\
07\end{array}$ & $\begin{array}{l}39 \\
47\end{array}$ & $\begin{array}{l}33.1 \\
12.6\end{array}$ & $\begin{array}{l}44.30 \\
43.80\end{array}$ & N. & $\begin{array}{l}110.92 \mathrm{~W} . \\
110.18 \mathrm{~W} .\end{array}$ & $\begin{array}{l}5 \\
5\end{array}$ & $\begin{array}{l}3.8 \\
\ldots .\end{array}$ & $\ldots$ & $\begin{array}{l}3.1 \mathrm{G} \\
3.2 \mathrm{G}\end{array}$ & $\begin{array}{l}\text { IV } \\
. .\end{array}$ & $\mathrm{G}$ & $\begin{array}{l}\text { MAR. } \\
\text { MAR . }\end{array}$ & $\begin{array}{r}7 \\
10\end{array}$ & $\begin{array}{ll}00 & \text { A.M. } \\
00 & \text { A.M. MST } \\
\text { MST }\end{array}$ \\
\hline
\end{tabular}

Table 2.-Summary of macroseismic data for U.S. earthquakes, January-March 1978

[Sources of the hypocenters and magnitudes: (A) Geophysical Institute, University of Alaska, Fairbanks; (B) University of California, Berkeley; (D) University of Montana, Missoula; (E) U.S. Department of Energy, Las Vegas, Nevada; (G) U.S. Geological Survey, National Earthquake Information Service; (H) U.S. Geological Survey, Hawaiian Volcano Observatory; (J) Weston Observatory, Massachusetts; (L) Lamont-Doherty Geological Observatory, Palisades, New York; (M) National Oceanic and Atmospheric Administration, Alaska Tsunami Warning Center, Palmer; (0) Earth Physics Branch, Seismological Service of Canada, Ottawa; (P) California Institute of Technology, Pasadena; (S) St. Louis University, St. Louis, Missouri; (T) University of Oklahoma, Leonard; (U) University of Utah, Salt Lake City; (V) Virginia Polytechnic Institute and State University, Blacksburg; (W) University of Washington, Seattle. Dates and origin times are listed in Universal Coordinated Time (UTC) giving the hour, minute, and second. Epicenters are shown in decimal degrees. only earthquakes with intensity data and explosions are listed]

Al abama

1 March (G) Northern Al abama Origin time: 040826.6 Epicenter: $\quad 34.42 \mathrm{~N} ., 86.61 \mathrm{~W}$. Depth: $\quad 5 \mathrm{~km}$ Magnitude: $2.5 \mathrm{mbLg}$ Intensity III: Huntsville area (V).

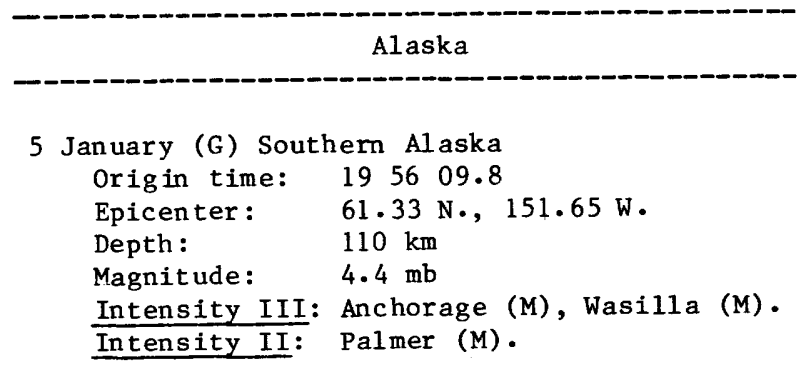


Table 2.--Summary of macroseismic data for U.S. earthquakes, January-March 1978

\section{Al aska--Continued}

6 January (G) Andreanof Islands, Aleutian Is lands Origin time: 070843.8

Epicenter: $\quad 51.78 \mathrm{~N} ., 176.01 \mathrm{~W}$.

Depth : $63 \mathrm{~km}$

Magnitude: $\quad 5.3 \mathrm{mb}$

Intensity IV: Adak (M).

6 January (G) Kenai Peninsula

Origin time: 215901.1

Epicenter: $\quad 60.91 \mathrm{~N} ., 149.38 \mathrm{~W}$.

Depth: $\quad 45 \mathrm{~km}$

Magnitude: $\quad 4.6 \mathrm{mb}, 4.9 \mathrm{ML}(\mathrm{M})$

Intensity V: Girdwood (small objects fell, not broken).

Intensity IV: Anchorage, Chugiak, Clam Gulch, Cooper Landing, Eagle River, Kenai, Ouzinkie, Seward, Soldotna, Whittier.

Intensity III: Elmendorf AFB, Moose Pass, Palmer.

9 January (G) Southern Alaska

Origin time: 070605.8

Epicenter: $\quad 62.00 \mathrm{~N} ., 148.82 \mathrm{~W}$.

Depth: $\quad 9 \mathrm{~km}$

Magnitude: $\quad 3.5$ ML (M)

Intensity III: Anchorage, Lower Susitna

Valley, Matanuska.

9 January (G) Andreanof Islands, Aleutian Islands Origin time: 221814.6

Epicenter: $\quad 51.61 \mathrm{~N} ., 177.17 \mathrm{~W}$.

Depth: $\quad 121 \mathrm{~km}$

Magnitude: $\quad 3.9 \mathrm{mb}$

Intensity II: Adak (M).

10 January (A) Central Alaska

Origin time: 120916.4

Epicenter: $\quad 64.74 \mathrm{~N} ., 147.44 \mathrm{~W}$.

Depth: $\quad 24 \mathrm{~km}$

Magnitude: 2.8 ML(M)

Intensity III: Fai rbanks.

18 January (G) Fox Is lands, Aleutian Is lands

Origin time: 170418.1

Epicenter: $\quad 52.92$ N., $166.43 \mathrm{~W}$.

Depth: $\quad 70 \mathrm{~km}$

Magnitude: None computed.

Intensity IV: Unalaska (M).

22 January (G) Cook Inlet

Origin time: 020254.0

Epicenter: $\quad 60.24 \mathrm{~N} ., 152.33 \mathrm{~W}$.

Depth :

Magnitude: None computed.

Intensity III: Anchorage (M).

27 January (G) Kenai Peninsula

Origin time: 185259.2

Epicenter: $\quad 60.37 \mathrm{~N} ., 151.12 \mathrm{~W}$.

Depth: $\quad 70 \mathrm{~km}$
Table 2.--Summary of macroseismic data for U.S. earthquakes, January-March 1978

\begin{tabular}{|c|c|}
\hline \multicolumn{2}{|r|}{ Alaska--Continued } \\
\hline & $\begin{array}{ll}\text { Magnitude: } & 4.7 \mathrm{mb} \\
\text { Intensity III: Anchorage (M), Eagle River } & (\mathrm{M}), \operatorname{Ken} a \mathrm{I}(\mathrm{M}), \text { Soldotna (M). }\end{array}$ \\
\hline 28 & $\begin{array}{ll}\text { January (G) Kenai Peninsula } \\
\text { Origin time: } & 185306.8 \\
\text { Epicenter: } & 60.07 \mathrm{~N} ., 151.33 \mathrm{~W} . \\
\text { Depth: } & 77 \mathrm{~km} \\
\text { Magnitude: } & 4.5 \mathrm{mb} \\
\text { Intensity III: } & \text { Kenai (M), Nikishka (M). } \\
\text { Intensity II: } & \text { Anchorage (M). }\end{array}$ \\
\hline 12 & $\begin{array}{ll}\text { February (G) Cook Inlet } \\
\text { Origin time: } & 085638.9 \\
\text { Epicenter: } & 59.45 \mathrm{~N} ., 152.62 \mathrm{~W} . \\
\text { Depth: } & 72 \mathrm{~km} \\
\text { Magnitude: } & 5.4 \mathrm{mb} \\
\text { Intensity V: } & \text { Anchor Point, Homer, Ouzinkie. } \\
\text { Intensity IV: Seldovia. } \\
\text { Intensity III: Kodiak, Kokhanok, Port Graham. } \\
\text { Intensity II: English Bay (M), Homer (M), } \\
\text { King Salmon (M). }\end{array}$ \\
\hline 16 & $\begin{array}{ll}\text { February }(G) \text { Southern Alaska } \\
\text { Origin time: } 205349.0 \\
\text { Epicenter: } \quad 61.31 \mathrm{~N} ., 144.89 \mathrm{~W} . \\
\text { Depth: } & \text { Normal. } \\
\text { Magnitude: } & 4.1 \mathrm{ML}(\mathrm{M}) \\
\text { Intensity IV: } & \text { Chitina. }\end{array}$ \\
\hline
\end{tabular}

6 March (G) Andreanof Islands, Aleutian Is lands Origin time: 184023.6

Epicenter: $\quad 51.76 \mathrm{~N} ., 175.81 \mathrm{~W}$.

Depth: $\quad 65 \mathrm{~km}$

Magnitude: $\quad 4.7 \mathrm{mb}$

Intensity II: Adak (M).

20 March (G) Southern Al aska

Origin time: 035905.0

Epicenter: $\quad 60.18 \mathrm{~N} ., 153.61 \mathrm{~W}$.

Depth :

$153 \mathrm{~km}$

Magnitude: $\quad 4.9 \mathrm{mb}$

Intensity II: Anchorage (M), Homer (M).

20 March (G) Southern Alaska

Origin time: $08 \quad 15 \quad 37.5$

Epicenter: $\quad 59.84 \mathrm{~N} ., 153.24 \mathrm{~W}$.

Depth : $\quad 134 \mathrm{~km}$

Magnitude: $\quad 3.8 \mathrm{mb}$

Intensity III: Soldotna (M).

Intensity II: Homer (M), Kenai (M).

31 March (G) Southern Alaska

Origin time: 003813.4

Epicenter: $\quad 61.77$ N., 151.41 W.

Depth :

Intensity V: Cooper Landing (hanging

pictures swung; buildings creaked),

Skwentna (hanging pictures swung; windows, 
Table 2.--Summary of macroseismic data for U.S. earthquakes, January-March 1978

Al aska--Continued

doors, dishes rattled; buildings creaked), Wasilla (hanging pictures swung; windows, doors, dishes rattled; buildings creaked).

Intensity IV: Anchorage (M), Chugiak, Girdwood, Kasilof, Kenai, Talkeetna, Tyonek, Whittier, Willow.

Intensity III: Elmendorf AFB, Homer (M), Moose Pass, Palmer (M).

\section{Arizona}

10 March (P) Baja California

Origin time: 203436.2

Epicenter: $\quad 32.33 \mathrm{~N} ., 115.03 \mathrm{~W}$.

Depth: $\quad 5 \mathrm{~km}$

Magnitude: $\quad 3.5 \mathrm{ML}$

Intensity III: Yuma (telephone report).

11 March (G) Baja California

Origin time: 235746.8

Epicenter: $\quad 32.26 \mathrm{~N} ., 115.12 \mathrm{~W}$.

Depth: $\quad 5 \mathrm{~km}$

Magnitude: $\quad 4.8 \mathrm{mb}, 5.0 \mathrm{ML}(\mathrm{P})$

This earthquake destroyed approximately 30 houses, cracked dozens of buildings, and interrupted electric power and telephone communications in the cities of San Luis, Sonora, and Luis Sanchez, Mexico.

Intensity VI: Arizona-Yuma (cracked plaster).

Intensity III: California--El Centro (press repor $t$ ).

12 March (G) Baja California

Origin time: 003015.7

Epicenter: $\quad 32.23 \mathrm{~N} ., 115.14 \mathrm{~W}$.

Depth: $\quad 5 \mathrm{~km}$

Magnitude: $\quad 4.5 \mathrm{mb}, 4.7 \mathrm{ML}(\mathrm{P})$

Intensity IV: Arizona--Yuma.

Intensity III: California--southern Imperial Valley (telephone report).

12 March (G) Baja California

Origin time: 184224.3

Epicenter: $\quad 32.26 \mathrm{~N} ., 115.11 \mathrm{~W}$.

Depth: $\quad 5 \mathrm{~km}$

Magnitude: $\quad 4.9 \mathrm{mb}, \quad 4.9 \mathrm{ML}(\mathrm{P})$

Intensity VI:

Arizona--Yuma (cracked plaster, cracked streets).

Intensity IV:

Arizona--Somerton .

Calif ornia--Imperial, Win terhaven .

16 March (P) Baja California

Origin time: 015110.1

Epicenter: $\quad 32.30 \mathrm{~N}, 115.12 \mathrm{~W}$.
Table 2.--Summary of macroseismic data for U.S. earthquakes, January-March 1978

Arizona--Continued

Depth: $\quad 5 \mathrm{~km}$

Magnitude: $\quad 4.2 \mathrm{mb}(\mathrm{G}), 4.2 \mathrm{ML}$

Intensity IV: Yuma.

\section{Californ ia}

4 January (B) Nor thern California

Origin time: 122302.7

Epicenter: $\quad 40.57$ N., $124.55 \mathrm{~W}$.

Depth: $\quad 29 \mathrm{~km}$

Magnitude: $\quad 3.5 \mathrm{ML}$

Intensity IV: Ferndale, Fortuna.

Intensity III: Eel River Valley.

6 January (B) Northern California

Origin time: 104244.9

Epicenter: $\quad 39.35$ N., 123.16 W.

Depth: $2 \mathrm{~km}$

Magnitude: $\quad 3.0 \mathrm{ML}$

Intensity $\mathrm{V}$ : Willits.

14 January (B) Nor thern California

Origin time: 154544.8

Epicenter: $\quad 38.92$ N., $122.92 \mathrm{~W}$.

Depth: $11 \mathrm{~km}$

Magnitude: $\quad 3.1 \mathrm{ML}$

Intensity III: Southern Lake County (press report).

15 January (B) Northern California

Origin time: 143826.1

Epicenter: $\quad 39.17 \mathrm{~N} ., 123.24 \mathrm{~W}$.

Depth: $2 \mathrm{~km}$

Magnitude: $\quad 3.1 \mathrm{ML}$

Intensity IV: Ukiah (press report).

Intensity III: Willits (press report).

17 January (B) Nor thern California

Origin time: 205912.6

Epicenter: $\quad 39.19 \mathrm{~N} ., 123.24 \mathrm{~W}$.

Depth: $2 \mathrm{~km}$

Magnitude: $\quad 2.8 \mathrm{MI}$

Intensity V: Ukiah (hairline crack in the wall, dishes rattled, buildings swayed--press report).

25 January (P) Southern California

Origin time: 104050.0

Epicenter: $\quad 34.32 \mathrm{~N} ., 118.33 \mathrm{~W}$.

Depth: $\quad 5 \mathrm{~km}$

Magnitude: $2.7 \mathrm{ML}$

Intensity III: La Crescenta, Sylmar.

28 January (B) Nor thern California

Origin time: 084902.5

Epicenter: $\quad 39.40 \mathrm{~N} ., 123.35 \mathrm{~W}$.

Depth :

Magnitude: $2.5 \mathrm{ML}$ (B)

Intensity V: Willits (furniture and small objects moved; people awakened; windows, doors, dishes rattled). 
Table 2.--Summary of macroseismic data for U.S. earthquakes, January-March 1978

California--Continued

31 January (P) Southern California

Origin time: 141810.0

Epicenter: $\quad 34.18 \mathrm{~N} ., 118.63 \mathrm{~W}$.

Depth: $\quad 6 \mathrm{~km}$

Magnitude: $2.1 \mathrm{ML}$

Intensity III: Canoga Park, Chatsworth.

6 February (P) Southern California

Origin time: 003925.8

Epicenter: $\quad 34.03 \mathrm{~N} ., 116.78 \mathrm{~W}$.

Depth: $\quad 6 \mathrm{~km}$

Magnitude: $\quad 3.1 \mathrm{ML}$

Intensity III: Palm Springs.

6 February (P) Southern California

Origin time: 010128.9

Epicenter: $\quad 34.03 \mathrm{~N} ., 116.78 \mathrm{~W}$.

Depth: $\quad 6 \mathrm{~km}$

Magnitude: $\quad 3.3 \mathrm{MI}$

Intensity III: Palm Springs.

6 February (P) Imperial Valley

Origin time: 125714.2

Epicenter: $\quad 33.25 \mathrm{~N} ., 115.57 \mathrm{~W}$.

Depth: $\quad 4 \mathrm{~km}$

Magnitude: $\quad 3.4 \mathrm{ML}$

Intensity $\mathrm{V}$ : Niland (many awakened; windows, doors, dishes rattled; buildings shook).

Intensity III: Brawley (telephone report).

7 February (B) Northern California

Origin time: 133922.7

Epicenter: $\quad 39.41 \mathrm{~N} ., 123.17 \mathrm{~W}$.

Depth: $\quad 5 \mathrm{~km}$

Magnitude: $\quad 2.7 \mathrm{ML}$ (B)

Intensity V: Willits (many awakened; small objects shifted; buildings shook; windows, doors, dishes rattled).

11 February (P) Imperial Valley

Origin time: 014530.5

Epicenter: $\quad 33.02 \mathrm{~N} ., 115.53 \mathrm{~W}$.

Depth: $\quad 7 \mathrm{~km}$

Magnitude: $\quad 2.5 \mathrm{ML}$

Intensity II: Brawley.

11 February (P) Imperial Valley

Origin time: 014551.0

Fpicenter: $\quad 33.02 \mathrm{~N} ., 115.53 \mathrm{~W}$.

Depth: $\quad 5 \mathrm{~km}$

Magnitude: $2.5 \mathrm{ML}$

Intensity II: Brawley.

11 February (P) Imperial Valley

Origin time: 022551.4

Epicenter: $\quad 33.02 \mathrm{~N} ., 115.53 \mathrm{~W}$.

Magnitude: $\quad 3.1 \mathrm{ML}$

Depth: $\quad 6 \mathrm{~km}$

Intensity III: Brawley.
Table 2.--Summary of macroseismic data for U.S. earthquakes, January-March 1978

California--Continued

13 February (P) Southern California

Origin time: 180406.3

Epicenter: $\quad 34.02 \mathrm{~N} ., 117.22 \mathrm{~W}$.

Depth: $\quad 6 \mathrm{~km}$

Magnitude: $\quad 2.9 \mathrm{ML}$

Intensity II: Redlands.

14 February (B) Northern California

Origin time: 163346.8

Epicenter: $\quad 38.42 \mathrm{~N} ., 122.66 \mathrm{~W}$.

Depth: $\quad 9 \mathrm{~km}$

Magnitude: $2.8 \mathrm{ML}$

Intensity II: Santa Rosa.

14 February (B) Northern California

Origin time: 200309.5

Epicenter: $\quad 38.42 \mathrm{~N} ., 122.66 \mathrm{~W}$.

Depth: $\quad 9 \mathrm{~km}$

Magnitude: $2.5 \mathrm{ML}$

Intensity II: Santa Rosa.

14 February (B) Northern California

Origin time: 210355.8

Epicenter: $\quad 40.20 \mathrm{~N} ., 124.00 \mathrm{~W}$.

Depth: $\quad 10 \mathrm{~km}$

Magnitude: $\quad 3.0 \mathrm{ML}$

Intensity IV: Rio Dell.

Intensity III: Ferndale, Fortuna.

23 February (P) Imperial Valley

Origin time: 164303.7

Epicenter: $\quad 32.82 \mathrm{~N} ., 115.60 \mathrm{~W}$.

Depth: $19 \mathrm{~km}$

Magnitude: $\quad 3.6 \mathrm{MI}$

Intensity V: Seeley (felt by all; windows, doors, dishes rattled; hanging pictures swung, hanging objects swung slightly). Intensity IV: Imperial.

1 March (P) Southern California

Origin time: 045431.2

Epicenter: $\quad 34.53 \mathrm{~N} ., 116.77 \mathrm{~W}$.

Depth: $\quad 5 \mathrm{~km}$

Magnitude: $\quad 4.4 \mathrm{ML}, 4.4 \mathrm{ML}$ (B)

Intensity VI: White Water (cracked plaster; light furniture shifted).

Intensity V: Colton, Fawnskin, Highland.

Intensity IV: Angelus Oaks, Apple Valley, Big Bear City, Bryn Mawr, Chino, Crestline, Etiwanda, Green Valley Lake, Montrose, Mount Baldy, Palomar Mountain, Rimforest, San Bernardino, Twin Peaks.

Intensity III: Barstow, Big Bear Lake, Crest Park, Del Rosa, Lake Arrowhead, Lucerne Valley, Newberry Springs, Norton AFB, Victorville (press report).

Intensity II: Azusa, Blue Jay, Burbank, Daggett, West Covina (press report), Wrightwood. 
Table 2.--Summary of macroseismic data for U.S. earthquakes, January-March 1978

Californ ia--Continued

8 March (P) Southern California

Origin time: 144934.9

Epicenter: $\quad 33.83 \mathrm{~N} ., 117.88 \mathrm{~W}$.

Depth: $\quad 5 \mathrm{~km}$

Magnitude: $\quad 2.9 \mathrm{MI}$

Intensity IV: Anaheim (Brookhurst Center, Sunkist), Fullerton, Long Beach, Orange, Placentia, Santa Ana, Westminster.

Intensity III: Brea (press report), Buena

Park (press report), Cypress (press

report), Garden Grove, La Mirada, Midway

City, Stanton, Sunny Hills, Tustin (press report), Yorba Linda.

11 March (G) Baja California

Origin time: 235746.8

See Arizona listing.

12 March (G) Baja California

Origin time: 003015.7

See Arizona listing.

12 March (G) Baja California

Origin time: 184224.3

See Arizona listing.

13 March (P) Southern California

Origin time: 163815.7

Epicenter: $\quad 33.93 \mathrm{~N} ., 117.98 \mathrm{~W}$.

Depth: $\quad 4 \mathrm{~km}$

Magnitude: $\quad 3.2 \mathrm{ML}$

Intensity VI: La Mirada (cracked plaster).

Intensity V: Buena Park, La Habra,

Surfside.

Intensity IV: Anaheim (press report),

Bellflower, Fullerton, Perry, Pico Rivera, Whittier.

Intensity III: Alhambra, Brea (press report), Compton, Man hat tan Beach, Rowland Heights. Intensity II: La Puente, Norwalk, Santa Ana.

14 March (P) Southern California

Origin time: 160951.2

Epicenter: $\quad 33.95 \mathrm{~N} ., 117.97 \mathrm{~W}$.

Depth: $\quad 4 \mathrm{~km}$

Magnitude: $\quad 2.5 \mathrm{ML}$

Intensity IV: La Habra.

Intensity III: Fullerton, Santa Fe Springs, Whittier.

15 March (P) Imperial Valley

Origin time: 040249.4

Epicenter: $\quad 32.78 \mathrm{~N} ., 115.53 \mathrm{~W}$.

Depth: $\quad 5 \mathrm{~km}$

Magnitude: $\quad 2.9 \mathrm{MI}$

Intensity III: El Centro, Imperial (telephone report).
Table 2.--Summary of macroseismic data for U.S. earthquakes, January-March 1978

Californ ia--Contin ued

15 March (P) Imperial Valley

Origin time: 040939.7

Epicenter: $\quad 32.78 \mathrm{~N} ., 115.57 \mathrm{~W}$.

Depth: $\quad 5 \mathrm{~km}$

Magnitude: $\quad 3.2 \mathrm{ML}$

Intensity III: E1 Centro, Imperial (telephone report).

20 March (P) Southern California

Origin time: 000756.7

Epicenter: $\quad 36.00 \mathrm{~N} ., 117.87 \mathrm{~W}$.

Depth: $\quad 1 \mathrm{~km}$

Magnitude: $\quad 3.0 \mathrm{ML}, 3.6 \mathrm{ML}$ (B)

Intensity III: Haiwee Reservoir (telephone report).

21 March (B) Northern California

Origin time: 140512.1

Epicenter: $\quad 40.44$ N., 124.45 W:

Depth: $\quad 11 \mathrm{~km}$

Magnitude: $\quad 3.5 \mathrm{ML}$

Intensity IV: Bayside, Loleta, Rio Dell,

Scotia.

Intensity III: Ferndale.

26 March (B) Northem California

Origin time: 002704.4

Epicenter: $\quad 39.19 \mathrm{~N} ., 123.20 \mathrm{~W}$.

Depth :

$10 \mathrm{~km}$

Magnitude: $\quad 4.9 \mathrm{mb}(\mathrm{G}), 4.4 \mathrm{ML}$

This earthquake was felt over an area of about $8,200 \mathrm{sq} \mathrm{km}$ centered in the Ukiah area (fig. 7 ).

Loss of about $\$ 10,000$ from glass bottles and containers being thrown to the floor and broken was sustained by grocery and 1 iquor stores in the Ukiah area. This earthquake triggered three accelerographs located at Coyote Dam near Ukiah (Porcella, 1978). The accelerographs are located at the center crest, center toe, and south abutment of the dam. Maximum acceleration recorded at the crest was $0.30 \mathrm{~g}$, at the toe was $0.34 \mathrm{~g}$, and at the south abutment was $0.20 \mathrm{~g}$.

The press reported damage at a new warehouse at the Parducci Winery south of Ukiah. Huge storage tanks in the warehouse, welded to plates on the floor to hold them rigid, contained 200 tons of liquid. The liquid moved and about 1,000 gallons spilled, damaging the tanks. Liquid in free-floating tanks in another building sustained minor spillage, but there was no damage to the tanks. The drop ceiling in the new warehouse was also damaged. 


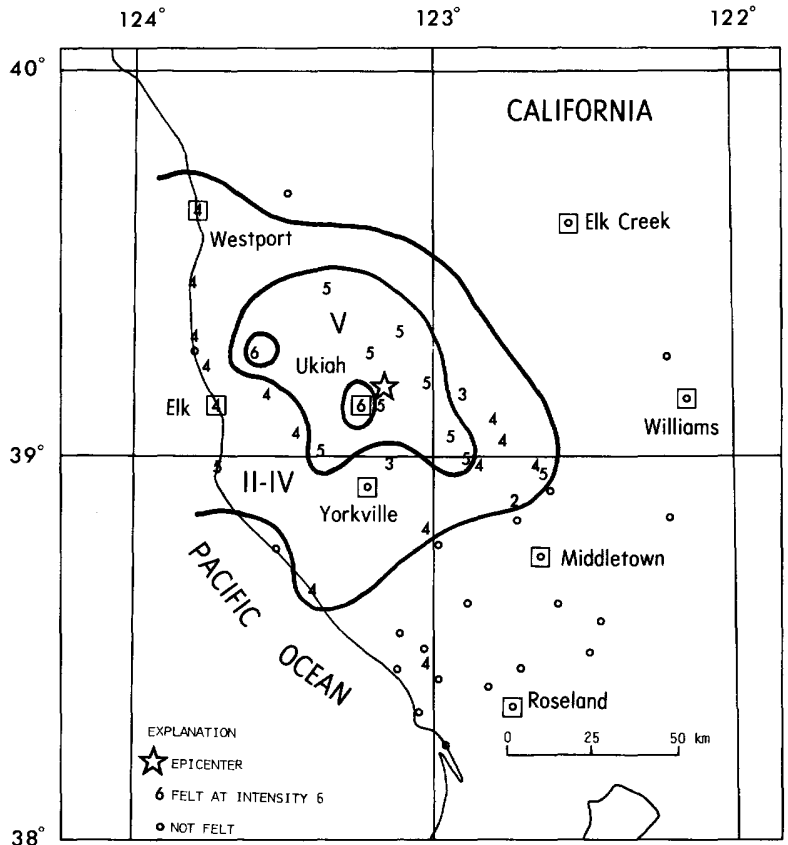

FIGURE 7.--Isoseismal map for the northern California earthquake of 26 March 1978, $002704.4 \quad$ UTC. Roman numerals represent Modified Mercalli intensities between isoseismals; Arabic numerals are use to represent these intensities at specific sites.

Table 2.--Summary of macroseismic data for U.S. earthquakes, January-March 1978

California--Continued

Intensity VI: Comptche (windows broken), Ukiah (pictures fell, glass bottles and jars broken in grocery and liquor stores, burglar alarm set off, damage to Parducci Winery).

Intensity V: Boonville, Clearlake Highlands, Finley, Kelseyville, Lakeport, Manchester, Potter Valley, Redwood Valley, Talmage, Willits, Witter Springs.

Intensity IV: Albion, Clearlake Park, Cloverdale, Elk, Fort Bragg, Glenhaven, Lucerne, Mendocino, Monte Rio, Navarro, Philo, Stewarts Point, Westport.

Intensity III: Hopland, Upper Lake. Intensity II: Loch Lomond.

26 March (B) Northern California

origin time: 003411.7

Epicenter: $\quad 39.22 \mathrm{~N} ., 123.18 \mathrm{~W}$.

Depth: $\quad 5 \mathrm{~km}$

Magnitude: $\quad 3.1 \mathrm{ML}$

Intensity IV: Talmage, Ukiah.

Intensity III: Willits.

26 March (B) Nor thern California

Origin time: 011909.7

Epicenter: $\quad 39.19$ N., 123.17 W.
Table 2.--Summary of macroseismic data for U.S. earthquakes, January-March 1978

California--Continued

$\begin{array}{ll}\text { Depth: } & 10 \mathrm{~km} \\ \text { Magnitude: } & 3.6 \mathrm{mb}(\mathrm{G}), 3.6 \mathrm{ML}\end{array}$

Intensity IV: Talmage, Ukiah.

Intensity III: Willits.

26 March (B) Northern California

Origin time: 022916.6

Epicenter: $\quad 39.20 \mathrm{~N} ., 123.23 \mathrm{~W}$.

Depth: $\quad 10 \mathrm{~km}$

Magnitude: $\quad 3.3 \mathrm{ML}$

Intensity IV: Talmage, Ukiah.

Intensity III: Willits.

26 March (B) Northern California

Origin time: $04 \quad 28 \quad 18.7$

Epicenter: $\quad 39.20 \mathrm{~N} ., 123.21 \mathrm{~W}$.

Depth: $\quad 10 \mathrm{~km}$

Magnitude: $\quad 3.5 \mathrm{ML}$

Intensity IV: Talmage, Ukiah.

Intensity III: Comptche, Willits.

27 March (B) Northern California

Origin time: 084403.0

Epicenter: $\quad 40.43 \mathrm{~N} ., 124.31 \mathrm{~W}$.

Depth: $14 \mathrm{~km}$

Magnitude: $\quad 3.2 \mathrm{ML}$

Intensity V: Rio Dell.

Intensity IV: Ferndale, Fortuna.

Intensity III: Scotia (press report), Weot1.

\section{Florida}

12 January Central Florida

Origin time: 2110

Epicenter: Not located.

Depth: None computed.

Magnitude: None computed.

Intensity IV: Haines City (press report).

Hawaii

The locations listed below that are followed by (H) designate intensity values assigned by the Hawaiian Volcano Observatory.

4 January (H) Island of Hawaii

Origin time: 184227.2

Epicenter: $\quad 19.33 \mathrm{~N} ., 155.18 \mathrm{~W}$.

Depth :

$10 \mathrm{~km}$

Magnitude: $\quad 3.7 \mathrm{ML}$

Intensity IV: Hilo (H).

Intensity III: Kurtistown $(\mathrm{H})$, Laupahoehoe (H), Volcano (H).

5 January (H) Is land of Hawaii

Origin time: 135846.6

Epicenter: $\quad 19.32 \mathrm{~N} ., 155.27 \mathrm{~W}$.

Depth: $\quad 10 \mathrm{~km}$

Magnitude: $\quad 3.2 \mathrm{ML}$

Intensity IV: Ainahou Ranch (H). 
Table 2.--Summary of macroseismic data for U.S. earthquakes, January-March 1978

\section{Hawaii--Continued}

11 January (H) Island of Hawaii

Origin time: 083311.3

Epicenter: $\quad 19.33 \mathrm{~N} ., 155.22 \mathrm{~W}$.

Depth: $\quad 10 \mathrm{~km}$

Magnitude: $\quad 3.8 \mathrm{ML}$

Intensity IV: Hilo (H).

Intensity III: Glenwood (H), Hamakua (H), Mountain View (H), Puna (H), Volcano (H).

13 January (H) Is land of Hawaii

Origin time: 003406.1

Epicenter: $\quad 19.53 \mathrm{~N} ., 155.95 \mathrm{~W}$.

Depth : $\quad 10 \mathrm{~km}$

Magnitude: $\quad 3.0 \mathrm{ML}$

Intensity III: Kona (H).

23 January (H) Is land of Hawaii

Origin time: 062138.5

Epicenter: $\quad 19.33 \mathrm{~N} ., 155.06 \mathrm{~W}$.

Depth: $\quad 9 \mathrm{~km}$

Magnitude: $\quad 3.7 \mathrm{ML}$

Intensity IV: Glenwood (H), Hilo (H),

Kalapana (H), Kurtistown $(\mathrm{H})$, Mountain View (H), Volcano $(\mathrm{H})$.

Intensity III: Papaikou (H).

23 January (H) Is land of Hawaii

Origin time: 062826.9

Epicenter: $\quad 19.34$ N., $155.07 \mathrm{~W}$.

Depth: $\quad 9 \mathrm{~km}$

Magnitude: $\quad 3.6 \mathrm{ML}$

Intensity IV: Glenwood (H), Hilo (H),

Kurtistown $(\mathrm{H})$, Mountain View $(\mathrm{H})$, Volcano

( $\mathrm{H})$.

Intensity III: Papaikou (H).

25 January (H) Island of Hawaii

Origin time: 011816.8

Epicenter: $\quad 19.33$ N., 155.10 W.

Depth: $9 \mathrm{~km}$

Magnitude: $\quad 3.2 \mathrm{MI}$

Intensity III: Hilo (H).

3 February (H) Is land of Hawaii

Origin time: 053245.8

Epicenter: $\quad 19.43$ N., $155.30 \mathrm{~W}$.

Depth: $16 \mathrm{~km}$

Magnitude: $\quad 3.5 \mathrm{ML}$

Intersity IV: Glenwood (H), Hilo (H),

Kurtistown $(\mathrm{H})$, Mountain View $(\mathrm{H})$, Volcano (H).

Intensity III: Honomu (H), Papaikou (H).

14 February (H) Island of Hawaii

Origin time: 200506.2

Epicenter: $\quad 19.40 \mathrm{~N} \cdot, 155.28 \mathrm{~W}$.

Depth: $\quad 15 \mathrm{~km}$

Magnitude: $\quad 3.4 \mathrm{ML}$

Intensity III: Hawaiian Volcano Observatory (H), Volcano $(\mathrm{H})$.
Table 2.--Summary of macroseismic data for U.S. earthquakes, January-March 1978

Hawaii-Continued

25 February (H) Is land of Hawaii

Origin time: 085527.2

Epicenter: $\quad 19.44 \mathrm{~N} \cdot, 156.32 \mathrm{~W}$.

Depth: $\quad 17 \mathrm{~km}$

Magnitude: $\quad 3.2 \mathrm{ML}$

Intensity III: Holualoa.

2 March (H) Island of Hawaii

Origin time: 105102.9

Epicenter: $\quad 19.33 \mathrm{~N} ., 155.22 \mathrm{~W}$.

Depth: $\quad 9 \mathrm{~km}$

Magnitude: $\quad 3.7 \mathrm{MI}$

Intensity IV: Hilo (H), Volcano (H).

21 March (H) Island of Hawaii

Origin time: 051325.4

Epicenter: $\quad 19.37 \mathrm{~N} \cdot, 155.42 \mathrm{~W}$.

Depth: $\quad 12 \mathrm{~km}$

Magnitude: $\quad 3.3 \mathrm{ML}$

Intensity III: Kukaiau Ranch.

\section{Maine}

4 January ( $\mathrm{J}$ ) Southwestern Maine

Origin time: 192810.8

Epicenter: $\quad 44.04$ N., $70.51 \mathrm{~W}$.

Depth: $\quad 0 \mathrm{~km}$

Magnitude: $\quad 3.2 \mathrm{mbLg}, 3.2 \mathrm{mbLg}(\mathrm{L})$

Intensity IV: Cisco (J), Naples (press report), 0tisfield (press report).

Intensity III: Harrison (J), Oxford (J), Poland $(\mathrm{J})$.

Intensity II: Bridgton $(\mathrm{J})$, Canton $(\mathrm{J})$, Johnson Hill $(\mathrm{J})$, West Poland $(\mathrm{J})$.

Massachusetts

24 March (G) Southwest of Bermuda Is lands

Origin time: 004238.2

Epicenter: $\quad 29.86$ N., 67.39 W.

Depth :

$22 \mathrm{~km}$

Magnitude: $\quad 6.1 \mathrm{mb}, 6.0 \mathrm{MS}, 6.1 \mathrm{MS}(\mathrm{B})$,

Intensity IV: $6.0 \mathrm{MS}(\mathrm{P})$

Massachusetts--New Bedford.

Intensity III:

Massachusetts--Boston (press report). North Carolina--Asheville (press report), Camp Lejune (press report), Hatteras (press report), Wake County (press report).

Mon tan $a$

14 January (G) Southwestern Montana Origin time: 165350.3 
Table 2.--Summary of macroseismic data for U.S. earthquakes, January-March 1978

$\begin{array}{ll} & \text { Montana-Continued } \\ \text { Epicenter: } & 44.77 \mathrm{~N} ., 111.94 \mathrm{~W} . \\ \text { Depth: } & 5 \mathrm{~km} \\ \text { Magnitude: } & 3.6 \mathrm{ML} \\ \text { Intensity II : Lake Village (Yellowstone } & \text { Lationa1 Park). }\end{array}$

Nevada

13 January (G) Southern Nevada

origin time: 033937.0

Epicenter: $\quad 39.42 \mathrm{~N} ., 117.60 \mathrm{~W}$.

Depth: $\quad 5 \mathrm{~km}$

Magnitude: $\quad 4.1 \mathrm{ML}(\mathrm{B})$

Intensity V: Austin.

14 February (G) Southern Nevada

Origin time: 043524.0

Epicenter: $\quad 39.63$ N., 117.18 W.

Depth : $\quad 5 \mathrm{~km}$

Magnitude: $\quad 4.4 \mathrm{mb}, 4.8 \mathrm{ML}$ (B)

Intensity V: Austin, Eureka, Round

Mountain, Yerington.

Intensity IV: Gabbs.

Intensity II: Manhattan.

23 February (E) Southern Nevada

Origin time: 170000.164

Epicenter: $\quad 37.12 \mathrm{~N} ., 116.06 \mathrm{~W}$.

Depth: $\quad 0 \mathrm{~km}$

Magnitude: $\quad 5.7 \mathrm{mb}(\mathrm{G}), 5.4 \mathrm{ML}(\mathrm{B})$

Nevada Test Site explosion "REBLOCHON" at $37^{n}$ $07^{\prime} 25.24^{\prime \prime}$ N., $116^{2} 03^{\circ} 49.79^{\prime \prime}$ W. , surface

elevation $1288 \mathrm{~m}$, depth of burial $658 \mathrm{~m}$.

5 March (G) Northern Nevada

Origin time: 224618.2

Epicenter: $\quad 38.94 \mathrm{~N} ., 118.03 \mathrm{~W}$.

Depth: $\quad 5 \mathrm{~km}$

Magnitude: $\quad 4.0 \mathrm{mb}, 4.6 \mathrm{ML}(\mathrm{B})$

Intensity V: Gabbs (small objects fell).

15 March (G) Western Nevada

Origin time: 030914.9

Epicenter: $\quad 38.35 \mathrm{~N} ., 118.22 \mathrm{~W}$.

Depth: $\quad 5 \mathrm{~km}$

Magnitude: $\quad 3.2 \mathrm{ML}$

Intensity IV: Gabbs, Luning, Mina.

23 March (E) Southern Nevada

Origin time: 163000.200

Epicenter: $\quad 37.10 \mathrm{~N} ., 116.05 \mathrm{~W}$.

Depth : $\quad 0 \mathrm{~km}$

Magnitude: $\quad 5.7 \mathrm{mb}(\mathrm{G}), 5.5 \mathrm{ML}(\mathrm{B})$

Nevada Test Site explosion "ICEBERG" at $37^{\circ}$ $06^{\circ} 06.39^{\prime \prime}$ W., $116^{\circ} 03^{\circ} 04.13^{\prime \prime}$ W., surface elevation $1266 \mathrm{~m}$, depth of burial $640 \mathrm{~m}$.
Table 2.--Summary of macroseismic data for U.S. earthquakes, January-March 1978

\section{New York}

18 February (0) Southern Quebec

Origin time: 144825.0

Epicenter: $\quad 46.35 \mathrm{~N} ., 74.11 \mathrm{~W}$.

Depth: $\quad 7 \mathrm{~km}$

Magnitude: $\quad 4.0 \mathrm{mb}(\mathrm{G}), 4.0 \mathrm{mbLg}$,

Intensity $\mathrm{V}$ :

$$
3.8 \mathrm{mbLg}(\mathrm{J})
$$

Quebec--Saint Donat de Montcalm region. Intensity IV:

New York-Rouses Point.

5 March (L) Southeastern New York

Origin time: 075325.6

Epicenter: $\quad 41.35 \mathrm{~N}, 74.15 \mathrm{~W}$.

Depth: $\quad 5 \mathrm{~km}$

Magnitude: $2.1 \mathrm{mbLg}$

Intensity III: Highland Mills (J).

Nor th Carolina

25 February (V) Nor thern North Carolina

Origin time: 035327.7

Epicenter: $\quad 36.19 \mathrm{~N} ., 79.30 \mathrm{~W}$.

Depth: $\quad 8 \mathrm{~km}$

Magnitude: $\quad 2.2 \mathrm{mbLg}$

Intensity IV: Burlington.

22 March (V) Northern North Carolina

Origin time: 155226.7

Epicenter: $\quad 36.20 \mathrm{~N} ., 81.73 \mathrm{~W}$.

Depth: $\quad 1 \mathrm{~km}$

Magnitude: $\quad 2.9 \mathrm{mbLg}$

Possible explosion (V).

24 March (G) Southwest of Bermuda Islands Origin time: 004238.2

See Massachusetts listing.

Ok lahoma

9 March (T) Southem Oklahoma

Origin time: 063051.2

Epicenter: $\quad 34.07 \mathrm{~N} ., 97.40 \mathrm{~W}$.

Depth: $\quad 5 \mathrm{~km}$

Magnitude: $\quad 2.5 \mathrm{mbLg}$

Intensity II: Simon area (T).

Tennessee

18 January (S) Western Tennessee

Origin time: 234626.1

Epicenter: $\quad 36.25 \mathrm{~N} ., 89.42 \mathrm{~W}$. 
Table 2.--Summary of macroseismic data for U.S. earthquakes, January-March 1978

Tennessee--Continued

Depth: $\quad 5 \mathrm{~km}$

Magnitude: $\quad 2.6 \mathrm{mbLg}$

Intensity III: Ridgely (Tennessee Earthquake Information Center).

\section{Texas}

2 March (G) Southwestern Texas

Origin time: 100452.7

Epicenter: $\quad 31.56 \mathrm{~N} ., 102.51 \mathrm{~W}$.

Depth: $11 \mathrm{~km}$

Magnitude: $\quad 3.5 \mathrm{ML}$

Intensity III: Kermit (telephone report).

\section{Utah}

28 February (U) Northern Utah

Origin time: 002006.5

Epicenter: $\quad 40.76 \mathrm{~N} ., 112.20 \mathrm{~W}$.

Depth: $\quad 14 \mathrm{~km}$

Magnitude: $2.7 \mathrm{ML}$

Intensity V: Salt Lake City.

Intensity IV: Magna.

Intensity III: Tooele.

Intensity II: Farmington.

9 March (U) Northern Utah

Origin time: 063051.8

Epicenter: $\quad 40.76 \mathrm{~N} ., 112.08 \mathrm{~W}$.

Depth: $\quad 9 \mathrm{~km}$

Magnitude: $\quad 3.3 \mathrm{ML}$

Intensity VI: Magna (cracks in the exterior

wa11 of fire station no. 2; windows broken, plaster and dry wall cracked).

Intensity V: Salt Lake City.

Intensity IV: Hooper, Murray.

Intensity III: Centerville (press report), Iincoln, Midvale.

9 March (U) Northern Utah

Origin time: 064620.0

Epicenter: $\quad 40.77$ N., $112.08 \mathrm{~W}$.

Depth: $\quad 7 \mathrm{~km}$

Magnitude: $2.5 \mathrm{ML}$

Intensity IV: Magna (press report).

13 March (U) Nor thern Utah

Origin time: 133543.4

Epicenter: $\quad 40.76 \mathrm{~N} ., 112.08 \mathrm{~W}$.

Depth: $13 \mathrm{~km}$

Magnitude: $2.8 \mathrm{ML}$

Intensity IV: Magna.

Intensity III: Bountiful (press report),

Granger (press report), Salt lake City

(Glendale area and University of Utah

Hospita1--press report).

Intensity II: Midvale.
Table 2.--Summary of macroseismic data for U.S. earthquakes, January-March 1978

\section{Virginia}

17 March (V) Southwestern Virginia

Origin time: 182634.5

Epicenter: $\quad 36.75 \mathrm{~N} ., 80.74 \mathrm{~W}$.

Depth: $\quad 7 \mathrm{~km}$

Magnitude: $\quad 2.8 \mathrm{mbLg}$

Intensity IV: Galax, Hillsville, Lambsburg,

Laurel Fork, Woodlawn.

Intensity III: Dugspur.

Intensity II: Austinville, Independence.

\section{Washington}

5 March (W) Puget Sound area

Origin time: 181335.9

Epicenter: $\quad 48.06 \mathrm{~N} ., 123.00 \mathrm{~W}$.

Depth: $\quad 57 \mathrm{~km}$

Magnitude: $\quad 4.0 \mathrm{mb}(\mathrm{G}), 3.3 \mathrm{ML}(\mathrm{G})$

Intensity IV: Nordland.

Intensity III:

British Columbia--Victoria (telephone report).

Washington--North Seattle (telephone report), Oak Harbor (telephone report).

Intensity II: Hansville.

11 March (W) Puget Sound area

Origin time: 155211.2

Epicenter: $\quad 47.42 \mathrm{~N} ., 122.71 \mathrm{~W}$.

Depth: $25 \mathrm{~km}$

Magnitude: $\quad 4.3 \mathrm{mb}(\mathrm{G}), 4.8 \mathrm{ML}, 3.2 \mathrm{MS}(\mathrm{G})$

This earthquake was felt over an area of $20,000 \mathrm{sq} \mathrm{km}$ of northwest Washington ( $\mathrm{fig}$. 8).

Intensity VI: Crystal Mountain Ski Resort (crack in rock and mortar wall at the Summit House; open beams supporting the roof moved 1-2 cm), Freel and (cracked plaster), Grotto (cracked plaster), Longbranch (cracked plaster and windows).

Intensity. V: Ashford, Bremerton, Centralia, Dockton, Enumclaw, Everett, Federal Way, Hobart, Issaquah, La Grande, Leavenworth, Olympia, Port Orchard, Poulsbo, Preston, Redondo, Ronald, Seabeck, Seattle, Snoqualmie Pass, South Colby, Tacoma.

Intensity IV: Aberdeen, Allyn, Anderson Island, Baring, Belfair, Blakely Island, Brinnon, Burley, Cinebar, Clearlake, Des Moines, East 0lympia, Eatonville, Fort Lewis (Gray Army Airfield), Fox Is land, Gig Harbor, Glenoma, Hadlock, Hoodsport, Indianola, Kapowsin, Kent, La Conner, Lakebay, Little Rock, Lyman, Lynnwood, McKenna, Medina, Milton, Mukilteo, Olalla, olga, Orting, Pacific, Parkland, Port 


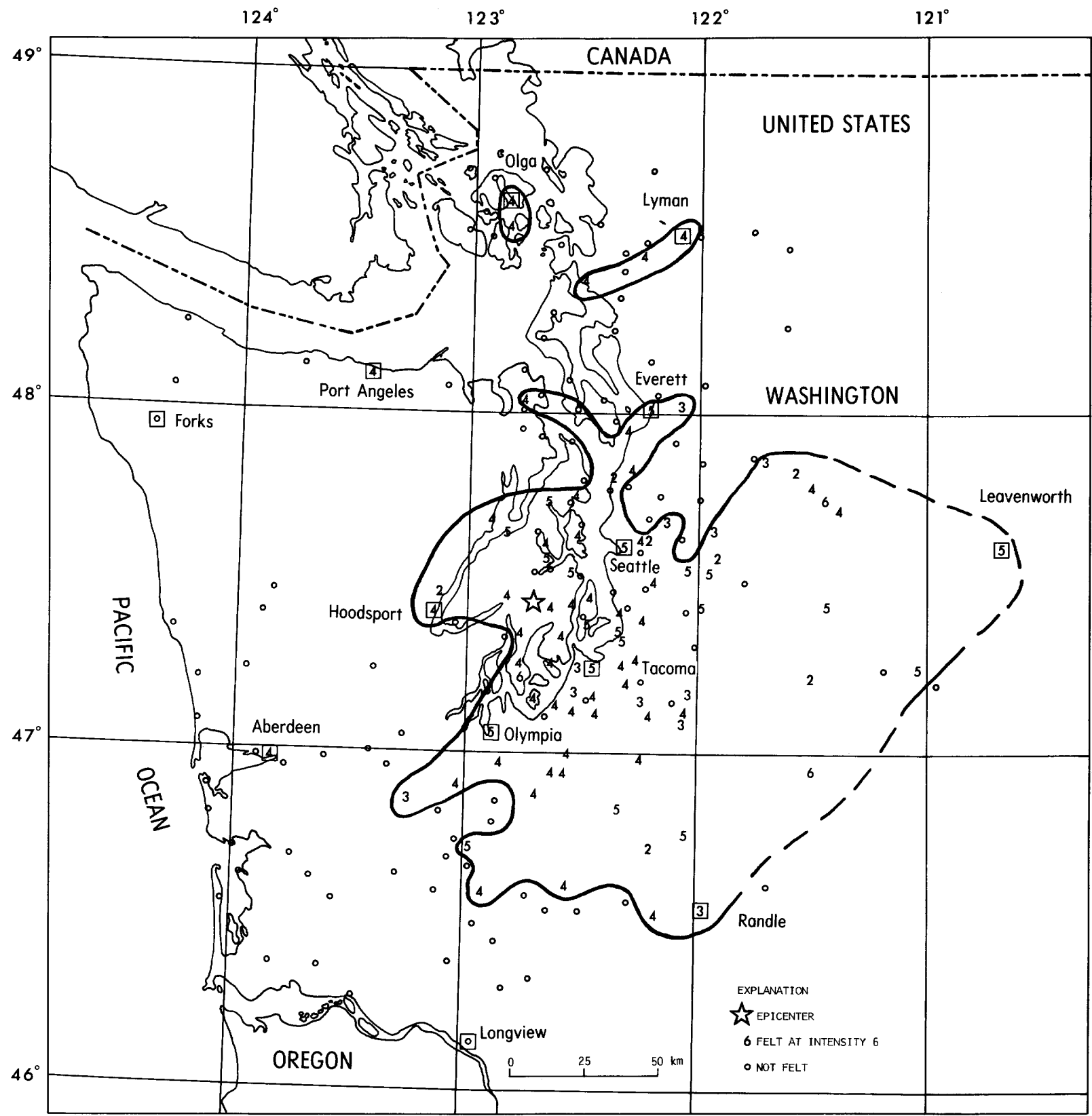

FIGURE 8.-Isoseismal map for the Puget Sound, Washington, earthquake of 11 March 1978 , 1552 11.2 UTC. Roman numerals represent Modified Mercalli intensities between isoseismals; Arabic numerals are used to represent these intensities at specific sites. Dashed line indicates the isoseismal is not well defined by the data.

Table 2.--Summary of macroseismic data for U.S. earthquakes, January-March 1978

Washington--Continued

Angeles, Proctor, Puyallup, Ranier, Renton, Roy, Skykomish, Spanaway, Tillicum,

Tracyton, Union, Vashon, Vaughn, Wilkeson,

Winslow, Yelon.
Table 2.--Summary of macroseismic data for U.S. earthquakes, January-March 1978

\section{Washington--Continued}

Intensity III: Buckley, Carbonado, Carnation, Fircrest, Fort Lewis, Gold Bar, Lake Stevens, Lakewood Center, McMillin, Napavine, Oakville, Randle, Redmond, 
Table 2.--Summary of macroseismic data for U.S. earthquakes, January-March 1978

Washington--Continued

Wallingford, Wenatchee (press report).

Intensity II: Bellevue, Edmonds, Fall City, Forest Park, Index, Lester, Lilliwaup, Mineral.

29 March (W) Northwest Washington

Origin time: 121638.4

Epicenter: $\quad 48.20 \mathrm{~N} ., 122.76 \mathrm{~W}$.

Depth: $\quad 24 \mathrm{~km}$

Magnitude: $\quad 2.7 \mathrm{ML}(\mathrm{G})$

Intensity IV: Oak Harbor.

31 March (W) Puget Sound area

Origin time: 080300.2

Epicenter: $\quad 47.42 \mathrm{~N} ., 122.72 \mathrm{~W}$.

Depth: $23 \mathrm{~km}$

Magnitude: $\quad 4.2 \mathrm{ML}(\mathrm{G})$

This earthquake was felt over an area of $7,000 \mathrm{sq} \mathrm{km}$ of the Puget Sound area (fig. 9).

Intensity V: Bremerton (hairline cracks in exterior walls), Brinnon, Chimacum, Hadlock, Manchester, Olalla, Port Orchard, Port Ludlow, Poulsbo, Quilcene, Rollingbay, Seabeck, Seattle, Tacoma, Tracyton, Union. Intensity IV: Auburn, Baring, Belfair, Bellevue, Burley, Crystal Mountain, Dockton, Fircrest, Fox Is land, Hansville, Index, Indianola, Kapowsin, Kingston, Lilliwaup, Matlock, Medina, Olympia, Puya1lup, Ravensdale, Tahuya, Vaughn. Intensity III: La Grande.

Intensity II: Carnation, Nordland.

Wyoming

13 January Yellowst one National Park

Seven earthquakes were felt in the eastern part of the park from January 13 to 21 . The largest magnitude of these quakes was about 1.8 ML. They were felt at Canyon Village and Lake, with the smaller magnitude events only being felt at lake. Maximum intensity was IV.

16 January (G) Southeast Wyoming

Origin time: 035003.1

Epicenter: $\quad 42.43 \mathrm{~N} ., 105.31 \mathrm{~W}$.

Depth: $\quad 5 \mathrm{~km}$

Magnitude: $\quad 3.0 \mathrm{ML}$

Intensity III: $14.5 \mathrm{~km}$ north of Laramie, Wheatland (telephone repor $t$ ).
Table 2.--Summary of macroseismic data for U.S. earthquakes, January-March 1978

Wyoming--Continued

2 February (G) Yellowstone National Park Origin time: 003625.6

Epicenter: $\quad 44.39 \mathrm{~N} ., 110.81 \mathrm{~W}$.

Depth: $\quad 5 \mathrm{~km}$

Magnitude: $\quad 3.6 \mathrm{mb}, 3.4 \mathrm{ML}, 3.6 \mathrm{ML}$ (D)

Intensity III: 01d Faithful. (Shocks at 00 4901.4 and 043025.9 with magnitudes of $2.8 \mathrm{ML}$ and 2.6 ML were also felt.)

2 February (G) Yellowst one National Park Origin time: 123556.2

Epicenter: $\quad 44.38 \mathrm{~N} ., 110.83 \mathrm{~W}$. Depth: $\quad 4 \mathrm{~km}$

Magnitude: $\quad 3.3 \mathrm{mb}, 3.1 \mathrm{ML}, 3.5 \mathrm{ML}$ (D)

Intensity III: 01d Faithful.

7 March (G) Yellowst one National Park

Origin time: 011047.6

Epicenter: $\quad 44.43 \mathrm{~N} ., 110.84 \mathrm{~W}$.

Depth: $\quad 5 \mathrm{~km}$

Magnitude: $\quad 3.7 \mathrm{mb}, 3.5 \mathrm{ML}, 3.8 \mathrm{ML}(\mathrm{D})$

From March 7 at 0109 UTC, to 1830 UTC, a swarm of 129 tremors was felt at old

Faithful. This series was known to have immediately induced minor changes in the thermal activity in the Upper Geyser Basin. These included increased turbidity in Heart Spring and Calida, and more vigorous splashing in Tea Kettle, with a rise in water 1 evel.

No changes in thermal activity occurred at the Norris Geyser Basin for 2.5 days after the swarm. But by March 23 Palpitar Spring had drained and the algae had died. At Steamboat Geyser minor eruptions appeared to have greater volume, and on March 28 the geyser had its first major eruption in 9 years. Runoff and sinter deposition at Cistun Springs had shifted from the west side of the pool to the southeast side, where two small trees were dying from the hot water (R. A. Hutchinson, National Park Service, written commun., 1978).

\section{Intensity V: 0ld Faithful.}

7 March (G) Yellowstone National Park Origin time: 073933.1

Epicenter: $\quad 44.30 \mathrm{~N} ., 110.92 \mathrm{~W}$. Depth: Magnitude: $\quad 3.8 \mathrm{mb}, 3.1 \mathrm{MI}$ Intensity IV: 0ld Faithful. 


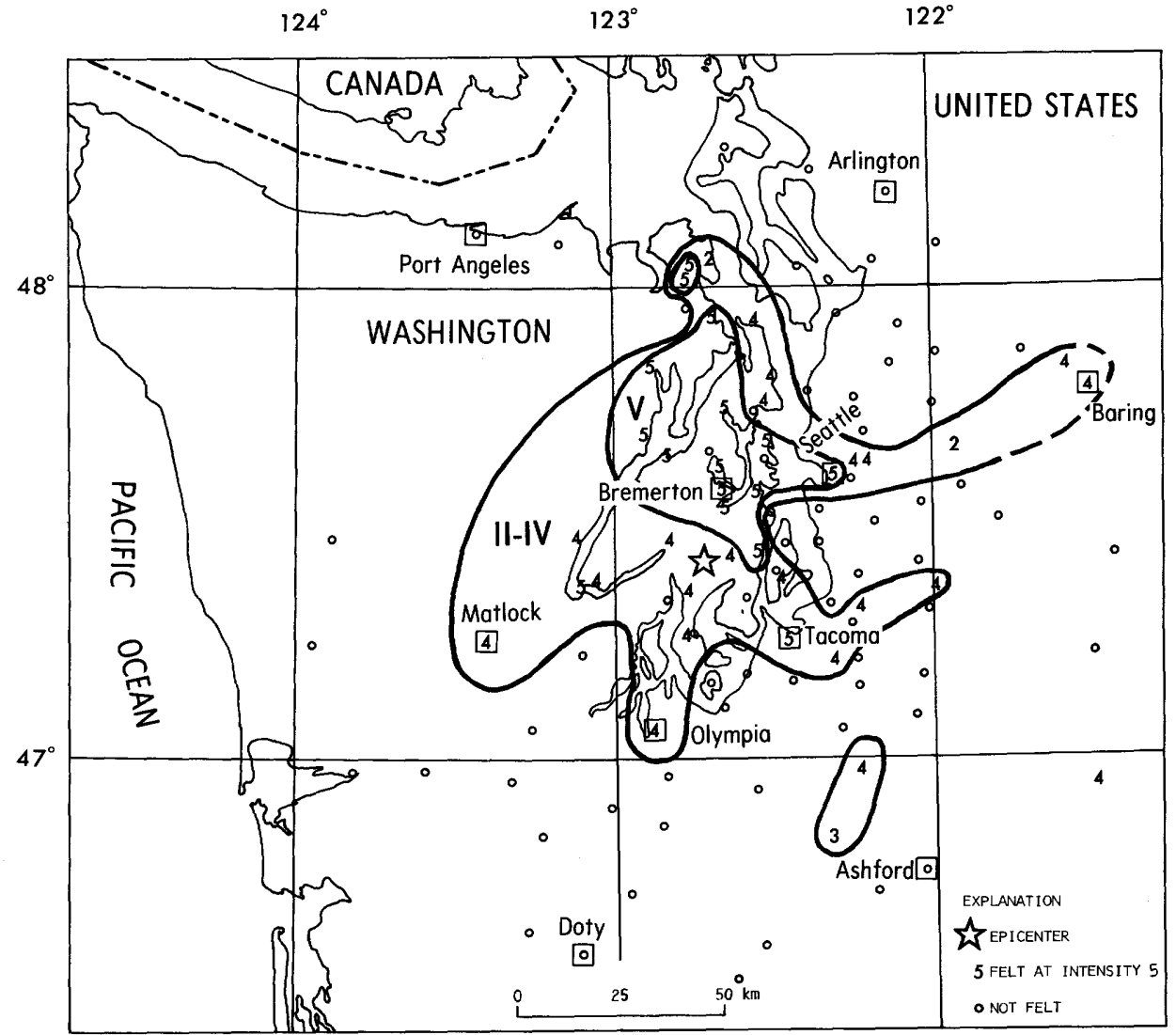

FIGURE 9.--Isoseismal map for the Puget Sound, Washington, earthquake of 31 March 1978. 080300.2 UTC. Roman numerals represent Modified Mercalli intensities between isoseismals; Arabic numerals are used to represent these intensities at specific sites. Dashed 1ine indicates the isoseismal is not well definded by the data.

\section{ACKNOWLEDGMENTS}

Listed below are the collaborators who furnished data to the National Earthquake Information Service for use in this Circular:

ALASKA:

CALIFORNIA:

HAWAII:

MASSACHUSETTS: Edward F. Chiburis, Weston

MISSOURI :
Staff of National Oceanic and Atmospheric Administration, Alaska Tsunami Warning Center, Palmer.

Clarence R. Allen, Seismological Laboratory, California Institute of Technology, Pasadena.

Bruce A. Bolt, Seismograph Station, University of California, Berkeley.

Robert Y. Koyanagi, U.S. Geological Survey, Hawaiian Volcano Observatory, Hawaii National Park. Observatory, Weston.

MONTANA:

NEW YORK :

OKLAHOMA:

UTAH :

VIRGINIA:

Ot to Nutt1i, Department of Geology and Geophysics, St. Louis University, St. Louis .
Anthony Qamar, University of Montana, Missoula.

Lynn R. Sykes and Yash P. Aggarwal, Lamont-Doherty Geological Observatory, Columbia University, Palis ades.

James E. Lawson, Jr., Earth Sciences Observatory, University of Oklahoma, Leonard.

Department of Geological and Geophysical Sciences, University of Utah, Salt Lake City.

G. A. Bollinger, Department of Geological Sciences., Virginia Polytechnic Institute and State University, Blacksburg.

WASHINGTON: Robert S. Crosson, Geophysics Program, University of Washington, Seattle.

Acknowledgment is also given to Paula Smith for aid in editing the manuscript, drafting the isoseismal maps, and preparing the computerized man us cript . 


\section{REFERENCES CITED}

Bath, Markus, 1966, Earthquake energy and magnitude, in Physics and chemistry of the Earth, Volume 7: Oxford and New York, Pergamon Press, p. 115-165.

Gutenberg, B. and Richter, C. F., 1956, Magnitude and energy of earthquakes: Annali di Geofisica, v. 9, no. 1, p. 1-15.

Nuttli, 0. W., 1973, Seismic wave attenuation and magnitude relations for eastern North
America: Journal of Geophysical Research, v . 78 , no. 5, p. 876-885.

Porcella, R. L., 1978, Recent strong-motion records, Seismic Engineering Program report, January-April 1978: U.S. Geological Survey Circular 785-A, p. 1 .

Richter, C. F., 1958, El ementary seismology: San Francisco, Calif., W. H. Freeman and Co., Inc., $768 \mathrm{p}$.

Wood, H. O., and Neumann, F., 1931, Modified Mercalli Intensity Scale of 1931: Bulletin of the Seismological Society of America, v . 21 , no. 4, p. 277-283. 



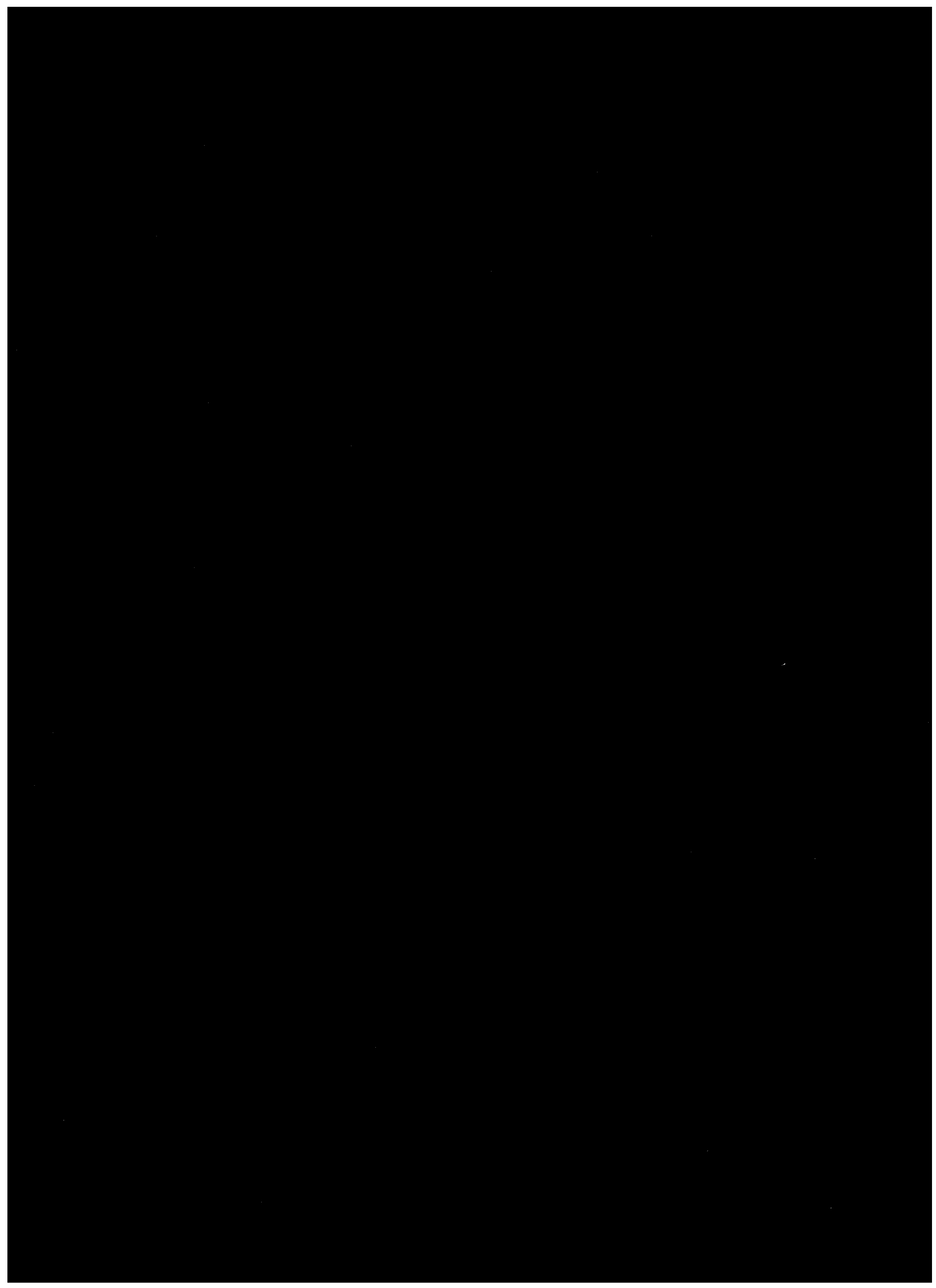




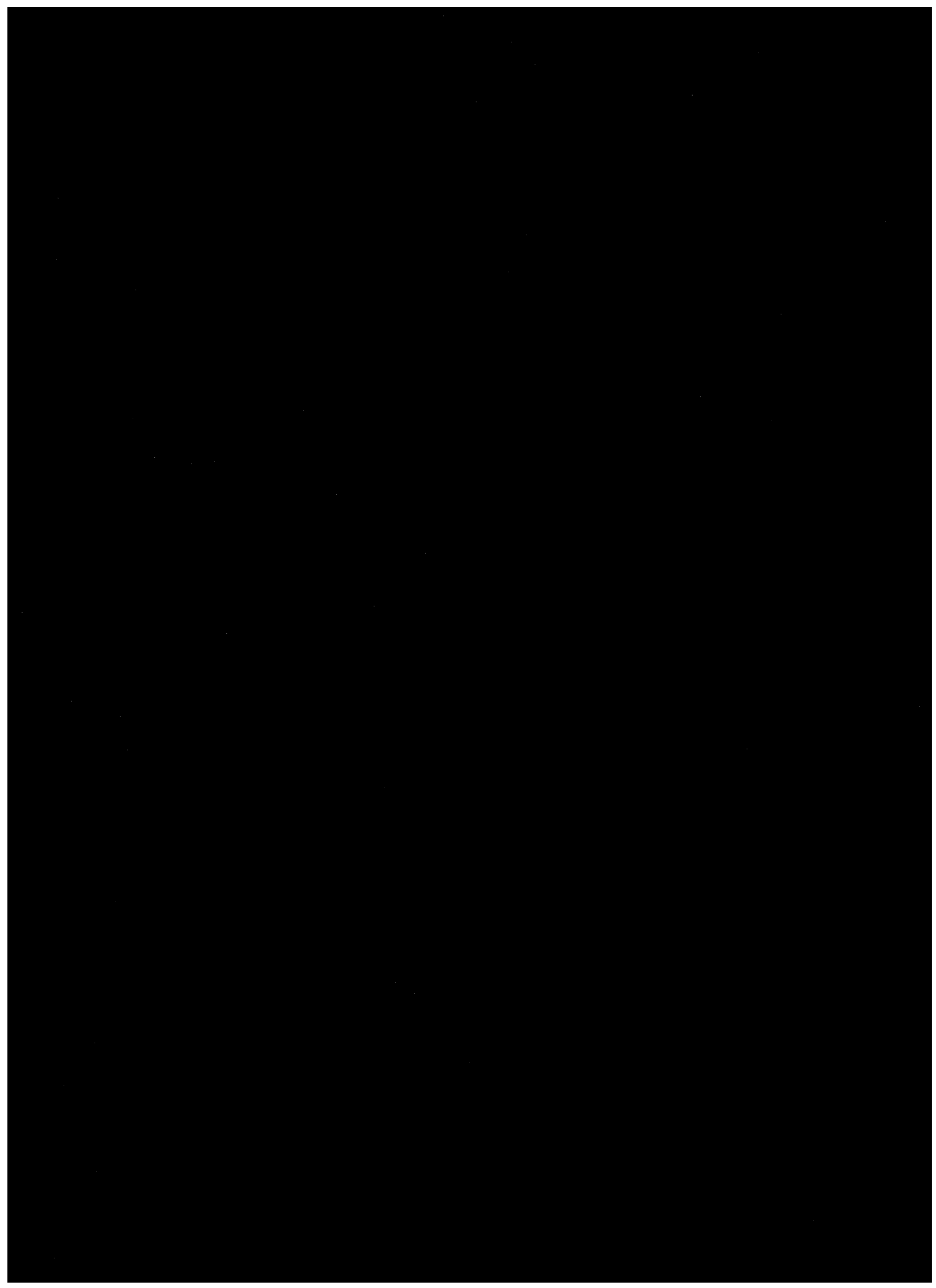

